\title{
General resonance mediation
}

\section{Moritz McGarrie}

Deutsches Elektronen-Synchrotron, DESY, Notkestrasse 85, 22607 Hamburg, Germany

E-mail: moritz.mcgarrie@desy.de

ABSTRACT: We extend the framework of general gauge mediation to cases where the mediating fields have a nontrivial spectral function, as might arise from strong dynamics. Demonstrating through examples, this setup describes a broad class of possible models of gauge mediated supersymmetry breaking. A main emphasis is to give general formulas for cross sections for $\sigma$ (visible $\rightarrow$ hidden $)$ in these resonance models. We will also give formulas for soft masses, A-terms and demonstrate the framework with a holographic setup.

KEywords: Supersymmetry Breaking, Supersymmetry and Duality, Supersymmetric Effective Theories

ARXIV EPRINT: 1207.4484 


\section{Contents}

1 Introduction 2

2 General resonance mediation $\quad 3$

2.1 The currents 3

$\begin{array}{lll}2.2 & \text { Minimal messenger sector } & 6\end{array}$

3 Currents and fields $\quad 6$

4 Examples $\quad 7$

4.1 Vector meson dominance/two site quiver 8

4.1.1 Old vector meson dominance versus quivers 9

4.2 A flat extra dimension 9

$\begin{array}{lll}4.3 & \text { General Regge-like Mediation } & 10\end{array}$

$\begin{array}{ll}\text { 4.3.1 From a soft wall model } & 11\end{array}$

$\begin{array}{lll}4.4 & \text { The unparticle limit } & 12\end{array}$

5 Scattering: a toy model $\quad \mathbf{1 3}$

$\begin{array}{lll}5.1 \text { A sum rule } & 14\end{array}$

$\begin{array}{llr}6 & \text { Scattering cross sections } & 15\end{array}$

$\begin{array}{lll}7 & \text { The A-term } & 19\end{array}$

8 Holography and the generating functional 20

8.1 Holographic gauge fields 21

$\begin{array}{ll}\text { 8.2 Soft masses and cross sections } & 23\end{array}$

$\begin{array}{lll}\text { 8.2.1 Soft masses in general } & 23\end{array}$

8.2.2 Soft masses with a minimal messenger sector 24

$\begin{array}{lll}\text { 8.2.3 Cross sections } & 25\end{array}$

$\begin{array}{ll}\text { 8.2.4 Messengers in the bulk } & 26\end{array}$

$\begin{array}{lll}8.3 & \text { Computing correlators of } \mathcal{O} & 27\end{array}$

$\begin{array}{ll}8.4 \text { A smoother transition? } & 27\end{array}$

9 Discussion and conclusion $\quad 27$ 


\section{Introduction}

Within the paradigm of gauge mediated supersymmetry breaking, perturbative models of supersymmetry breaking and perturbative mediation have been well understood and well mapped out [1]. One of the key challenges, if supersymmetry breaking is also to be a solution of the hierarchy problem, is that supersymmetry is broken dynamically [2], and hence the supersymmetry breaking sector is strongly coupled.

The formalism of general gauge mediation [3-6] encodes the effects of supersymmetry breaking in a set of current correlators. It assumes that when a set of parameters $\left(\left\{\alpha_{i}\right\},\left\{\lambda_{j}\right\}\right) \rightarrow 0$, the supersymmetric standard model and the sector that breaks supersymmetry utterly decouple from each other. One can then work perturbatively in these parameters. This framework is necessary to encode the effects of a breaking sector that is strongly coupled. Further, through using duality transformations and techniques of effective field theory, it may be possible to find a perturbative description which would give the same structure for the current correlators, for instance.

Whilst the programme is quite general, it is often useful to classify models by making assumptions about the type of theory one is analysing. In this paper we will assume that when the gauge fields of the standard model interact with the supersymmetry breaking sector their two point functions develop a non trivial spectral function. In other words the assumption is made that vector mesons participate in the mediation. Whilst this is naturally the case in five dimensional models [7-12], and these will be used as examples, it is hoped that these results will have application for theories that are four dimensional and strongly coupled.

Taking our motivation from the hadronic world, it is observed that at high energies photons behave like hadrons, except for their weaker coupling [13-15]. In particular there are intermediate resonances in photon-hadron interactions. Building on this one may expect that when standard model gauge fields interact with a strongly coupled supersymmetry breaking sector it may interact via resonances. One key focus will be to display this feature in scattering cross sections of visible $\rightarrow$ hidden processes.

Central to this point of view is the question of how one should think about the breaking sector when it is strongly coupled. Our perspective is that whilst one should expect messenger fields coupled to a supersymmetry breaking spurion [16] (which, in this analogy, we wish to loosely equate to the pion sector of QCD) more fundamentally, from the perspective of gauge interactions, there will also be a vast array of resonances, many with the same quantum numbers as the standard model gauge fields. This suggests not only new vector mesons in $\mathrm{U}(1)_{\mathrm{em}}$ but also color octets or "colorons" and "colorinos" of $\mathrm{SU}(3)_{c}[17,18]$ as the first signals of a supersymmetry breaking sector.

The resonances are bound states of hidden sector fields with the same quantum numbers as the standard model gauge fields. The resulting non trivial spectral function of the gauge fields will determine the form factor. The form factor, in this case, is a vector meson pole saturated charge distribution describing the interaction of external gauge fields with a strongly coupled sector. 
The form factor associated with the resonances encodes the low energy physics. Additionally by constructing a tower of continually broadening resonances one may observe cross sections with a rather smooth scaling property [19] to higher energies.

Whilst particular models will predict the form of the spectral function, ideally one would wish to fit the spectral function from observation, through measuring cross sections [20] such as those of section 6 . In this sense our approach will be mostly "bottom-up": we wish to extract soft masses, scattering cross sections and also to classify models from the behaviour of the spectral function associated with the resonances rather than specify a fully complete and perhaps overly complicated model from which one then attempts to derive these spectral functions. At some points however, the AdS/CFT perspective is used in which one should associate the four dimensional form factor and spectral function as arising from a gauge field propagating in a higher dimensional space.

In a general way, this paper will give results for soft terms and scattering cross sections of visible matter to supersymmetry breaking fields. Where it is useful, it will give examples of models that demonstrate key features of this rather broad class of model. A U(1) toy model of gauge mediation is used in general, although these results might be applicable for all of the $\mathrm{SU}(3) \times \mathrm{SU}(2) \times \mathrm{U}(1)$ gauge groups of the supersymmetric standard model.

Whilst completely compatible with four dimensional general gauge mediation, in addition the key features are additional standard model charged resonances arising from the supersymmetry breaking sector and light sfermions relative to gauginos at the high scale $M$.

\section{General resonance mediation}

In this section the currents that encode supersymmetry breaking effects from fields of the supersymmetry breaking sector are introduced. These current are extracted from a global symmetry of the supersymmetry breaking sector that is weakly gauged.

These currents will be used to derive general formulas for MSSM soft masses and also to compute scattering cross sections.

\subsection{The currents}

The supersymmetry breaking effects are encoded by introducing a real linear,

$$
D^{2} \mathcal{J}=\bar{D}^{2} \mathcal{J}=0
$$

global current supermultiplet $\mathcal{J}$

$$
\mathcal{J}=J+i \theta j-i \bar{\theta} \bar{j}-\theta \sigma^{\mu} \bar{\theta} j_{\mu}+\frac{1}{2} \theta^{2} \bar{\theta} \bar{\sigma}^{\mu} \partial_{\mu} j-\bar{\theta}^{2} \theta \sigma^{\mu} \partial_{\mu} \bar{j}-\frac{1}{4} \theta^{2} \bar{\theta}^{2} \square J .
$$

The currents are associated with a global symmetry of the supersymmetry breaking sector. The current correlators of which are defined in general gauge mediation [3]

$$
\begin{aligned}
i \tilde{C}_{0}\left(p^{2} / M^{2} ; M / \Lambda\right) & =\int \frac{d^{4} x}{(2 \pi)^{4}}\langle J(x) J(y)\rangle e^{i k .(x-y)} \\
i \Pi_{\mu \nu} \tilde{C}_{1}\left(p^{2} / M^{2} ; M / \Lambda\right) & =\int \frac{d^{4} x}{(2 \pi)^{4}}\left\langle j_{\mu}(x) j_{\nu}(y)\right\rangle e^{i k .(x-y)}
\end{aligned}
$$




$$
\begin{aligned}
i \sigma_{\alpha \dot{\alpha}}^{\mu} p_{\mu} \tilde{C}_{1 / 2}\left(p^{2} / M^{2} ; M / \Lambda\right) & =\int \frac{d^{4} x}{(2 \pi)^{4}}\left\langle j_{\alpha}(x) \bar{j}_{\dot{\alpha}}(y)\right\rangle e^{i k .(x-y)} \\
\epsilon_{\alpha \beta} M \tilde{B}\left(p^{2} / M^{2}\right) & =\int \frac{d^{4} x}{(2 \pi)^{4}}\left\langle j_{\alpha}(x) j_{\beta}(y)\right\rangle e^{i k .(x-y)}
\end{aligned}
$$

with $\Pi_{\mu \nu}=\left(p^{2} \eta^{\mu \nu}-p^{\mu} p^{\nu}\right)$. The scale $M$ is a typical mass scale of the hidden sector. The scale $\Lambda$ is a UV scale to regulate the integrals, which cancels in the "supertraced" combination that appears in scalar mass formulas:

$$
\Omega\left(\frac{p^{2}}{M^{2}}\right)=\left[\tilde{C}_{0}\left(\frac{p^{2}}{M^{2}}\right)+3 \tilde{C}_{1}\left(\frac{p^{2}}{M^{2}}\right)-4 \tilde{C}_{1 / 2}\left(\frac{p^{2}}{M^{2}}\right)\right] .
$$

Weakly gauging a global symmetry of the supersymmetry breaking sector, it is associated with it a set of interactions

$$
\mathcal{L} \supset g^{\prime} \int d^{4} \theta \mathcal{J}_{\text {SUSY }} \mathcal{V}^{\prime}
$$

This is a source term appearing in the generating functional. In full generality one may also introduce a source term for the supersymmetric standard model gauge symmetry

$$
\mathcal{L} \supset g \int d^{4} \theta \mathcal{J}_{\mathrm{SSM}} \mathcal{V}
$$

Where the $\mathcal{V}$ and $\mathcal{V}^{\prime}$ are real $\mathcal{V}=\mathcal{V}^{\dagger}$ vector superfields which in components are given by

$$
\mathcal{V}=-\theta \sigma^{\mu} \bar{\theta} A_{\mu}-i \theta^{2} \bar{\theta} \bar{\lambda}+i \bar{\theta}^{2} \theta \lambda+\frac{1}{2} \theta^{2} \bar{\theta}^{2} D
$$

In complete generality the vector fields and couplings in eq. (2.8) and eq. (2.9) may differ in the bare action. One only requires that for instance

$$
\frac{i \delta^{2} \log Z\left[\left\{\mathcal{J}_{i}\right\}\right]}{\delta j_{\text {SUSY }}^{\mu}(x) \delta j_{\text {SSM }}^{\nu}(y)}=\left\langle A_{\mu}(x) A_{\nu}^{\prime}(y)\right\rangle
$$

defines a meaningful two point function.

In models for which one has a perturbative description, this spectral function arises quite often: The spectral function encodes the overlap of the fields directly coupling to the supersymmetry breaking sector, with the gauge fields of the supersymmetric standard model. In Deconstructed models the spectral function arises in summing over mass eigenstates in the two point function of interaction eigenstates of the lattice [11,21]. In warped $5 \mathrm{~d}$ models it coincides with the Kaluza-Klein states or resonances of the bulk theory [8] or when fields depend on an additional parameter [22]. Examples in the next sections will show that to model vector meson dominance and its generalisations requires that the supersymmetry breaking currents do not directly couple to external fields but instead to intermediate resonances in the weakly coupled dual description.

In this paper it is assumed that it is a general consequence of the strong dynamics of the supersymmetry breaking sector. Within gauge mediation, one could hope that every mediating sector could be described in terms of a specified spectral function. 
Assuming that the two point function for the mediating gauge fields between each set of sources is given by a general Källén-Lehmann spectral representation,

$$
\int d^{4} x e^{i p . x}\left\langle A_{\mu}(x) A_{\nu}^{\prime}(0)\right\rangle=\int d \sigma^{2} \frac{i \rho(\sigma) \Pi^{\mu \nu}\left(p^{2}\right)}{p^{2}\left(p^{2}-\sigma+i \epsilon\right)}
$$

Following the Feynman prescription, which will be dropped from the notation henceforth. The $\rho\left(\sigma^{2}\right)$ is a spectral function. Multiplying by $p^{2}$, a convention has been chosen in which the function is dimensionless. In this way one may derive general results which may be applicable to particular models by specifying the exact form the spectral function. Additional poles in the spectral function may be thought of as composite particles as these do not have associated fields in the Lagrangian. The use of effective actions will allow us to determine these poles as they have an associated field in the effective description.

If one considers the mediating fields to be described by the spectral representation eq. (2.12), then the scalar soft masses are given by

$$
m_{\phi}^{2}=-(4 \pi \alpha)^{2} \int \frac{d^{4} p}{(2 \pi)^{4}} \frac{1}{p^{2}} \int d \sigma^{2} \int d \sigma^{\prime 2} \frac{\rho\left(\sigma^{2}\right)}{p^{2}-\sigma^{2}} \frac{\rho\left(\sigma^{\prime 2}\right)}{p^{2}-\sigma^{\prime 2}} \Omega\left(\frac{p^{2}}{M^{2}}\right) .
$$

to leading order in $\alpha_{S M}$. Assuming a $\mathrm{U}(1)$ toy model, ignoring group factors and taking $\rho_{a}=\rho$ for all $a=0,1 / 2,1$. There is a typical mass scale of the theory $M$, which for perturbative models is the messenger mass scale.

In eq. (2.13) two spectral integrals have been explicitly pulled out from inside the correlators, associated with the outer loop of the typical two-loop diagrams. The expression above is equivalent to that of general gauge mediation [3] in the presence of a "modified current operator" [23]. The outer loop will obtain corrections at higher order in $\alpha_{S M}$ [24]. We wish to stress that in using the spectral function we are not simply referring to the sub leading corrections in $\alpha_{S M}$ that arise quite normally in perturbation theory, but are instead referring to the spectral function that may arise from interacting with the strongly coupled hidden sector.

Despite the appearance of a spectral function, one may still expect a decoupling of the visible and hidden sector as $\alpha_{S M} \rightarrow 0$. Additionally for perturbative theories one may expect to return to a trivial spectral function

$$
\lim _{s \rightarrow \infty} \rho(s)=s \delta\left(\sigma^{2}\right),
$$

where $s=p^{2}$, for which one obtains the results of general gauge mediation [3].

The factorising form of the scalars masses is also observed in semi-direct gauge mediation [25] and in this case may be thought of as descending from the factorised form of the generating functional that defines the model in question. A similar result is demonstrated for holographic mediation models in section 8 .

The gaugino masses will be given by another current correlator eq. (2.6)

$$
m_{\lambda n, m}=(4 \pi \alpha) M \tilde{B}_{1 / 2}(0) f_{n} f_{m}
$$

where $f_{n}$ are some functions determined by the particular model. As the gaugino mass is evaluated at $p^{2}=0$ it is unnaffected by the form factors that effect the sfermion masses and cross sections. 
As models of this type fit within the framework of general gauge mediation, these models typically demonstrate the same features, that being flavour universality, compatibility with gauge unification, a gravitino or multiple pseudo-goldstini as LSPs with standard model superpartner NLSP and small A-terms. There are also certain sfermion sum rules [3]. In addition one expects light sfermion masses relative to gaugino masses at the high scale $M$.

\subsection{Minimal messenger sector}

Throughout this paper we will display both general results for an unspecified supersymmetry breaking sector and additional results based on the minimal messenger sector, which we use as a test model. The superpotential for the minimal messenger sector is given by

$$
W=X \Phi \tilde{\Phi}
$$

$X$ is a spurion or goldstino multiplet $X=M+\theta^{2} F$ and $\Phi, \tilde{\Phi}$ are messenger fields charged under a global symmetry which we associated with eq. (2.8). The mass eigenstates are two complex scalars $\phi_{+}, \phi_{-}$with masses $M^{2} \pm F$ and two 2-component fermions $\psi, \tilde{\psi}$ with mass $M$. For clarity, the hidden sector is comprised of $X, \Phi, \tilde{\Phi}$ for which the fields $\Phi, \tilde{\Phi}$ are messengers. The messenger fields should not be confused with the mediating sector, which are the gauge fields and resonances.

In such a model it is straightforward to determine the gaugino mass from eq. (2.15)

$$
m_{\lambda}=2 \frac{\alpha}{4 \pi} \frac{F}{M} g(x)
$$

where for $x=F / M^{2}$ where for $x<1, g(x) \sim 1$.

It will also be useful in this paper to evaluate the "super-traced" combination of current correlators eq. (2.7) in the limit $p^{2} / M^{2} \rightarrow 0$ found in [10]

$$
\lim _{p^{2} \rightarrow 0} \Omega\left(p^{2} / M^{2}\right)=\frac{-1}{(4 \pi)^{2}} \frac{2}{3} x^{2} h(x)
$$

where $h(x) \sim 1$ for $x<1$. It is independent of $p$ and will allow one to carry out the momentum integral on the form factors alone.

\section{Currents and fields}

In this section we wish to discuss some features relating vector meson dominance model and currents, which will be useful for understanding the relationship between different examples and in particular the relationship between VDM and $A d S_{5}$ models.

When one has a perturbative description and a lagrangian one may extract currents, for instance $j^{\mu}(x)=\bar{q} \gamma^{\mu} q(x)$, from Noether's theorem. The currents obey a current algebra and this arises from the commutators or anticommutators of the fields $\bar{q}, q$ in the current. Without a lagrangian one may apply this procedure in reverse and write a set of equal time commutators and Schwinger terms for some currents and construct a "field current identity" [23, 26-28]:

$$
j_{\mu}^{a}(x)=-C \rho_{\mu}^{a}(x)
$$


where $\rho_{\mu}$ is a general vector field which we will later associate with a massive vector meson. $\alpha$ is a group index and $\mathrm{C}$ is some so far undetermined coefficient. In fact this C may be determined [29] $C=m_{\rho}^{2} / g_{\rho}$ from a dispersion relation for the form factor $F\left(q^{2}\right)$ where $F(0)=1$. The field current identity is possible whenever $\rho_{\mu}$ arises from a gauge field of a hidden local symmetry [30]. In addition we should make some criteria: Normally Universality of the coupling $g_{\rho}$ to all hadronic currents arises as a result of current conservation [31]. Universality should be implemented in the interaction basis which may differ substantially from the mass basis. For a small but explicit breaking of the symmetry $b \mathcal{L}_{B}$ the charges of the symmetry will still relate physical states that are dominated by the state corresponding to exact preservation of this symmetry.

In a supersymmetric theory we may extend this to a "supercurrent field identity" which is a real linear multiplet

$$
\mathcal{J}_{\rho}=-\frac{m_{\rho}^{2}}{g_{\rho}} \rho \quad \text { where } \quad D^{2} \rho=\bar{D}^{2} \rho=0,
$$

which we may couple to

$$
g_{S M} \int d^{4} \theta \mathcal{J}_{\rho} V_{\text {external }} .
$$

If one is to interpret the vector meson as a gauge field of a local symmetry, we obtain a notion of a hidden local symmetry that is emergent. This notion of dominance may be generalised in which the form factor is saturated by a tower of meson poles. This has the appearance of a two point function of Kaluza-Klein modes of a bulk to boundary propagator:

$$
F\left(q^{2}\right) \sim \sum_{n} \frac{m_{n}^{2}}{g_{v}} \frac{F_{n}^{2}}{q^{2}-m_{n}^{2}} g_{n \phi \tilde{\phi}}
$$

and provide some generalised dispersion sum rules from $F(0)=1$. To write a gauge invariant and Lorentz invariant effective lagrangian to describe the features of a tower of massive spin one fields, requires a Higgs mechanism to generate masses for the gauge fields. Choosing then a quiver or extra dimension ${ }^{1}$ to generate the tower of states. These features may be generalised to $N$ local symmetries: $H L S_{N}$. Mediation between two boundaries on a "slice of AdS" generalises this notion to a continuum. The corresponding fields of $\mathcal{N}=1$ SYM in 5d that fill the supercurrent field identity are

$$
\mathcal{J}_{\rho} \supset\left(A^{\mu}, \lambda^{\alpha}, \bar{\lambda}^{\dot{\alpha}}, D_{5} \Sigma\right) .
$$

As $\mathcal{J}_{\rho}$ is a set of operators, the field current identity is an operator-field correspondence from the perspective of AdS/CFT.

\section{Examples}

It is useful to compare some typical examples of models that exhibit the features of resonance mediation. In this section we will complete a (not exhaustive) survey of different concrete models which realise such a scenario.

\footnotetext{
${ }^{1}$ Kaluza-Klein modes in $\mathcal{N}=15 \mathrm{~d}$ SYM also receive their masses from a supersymmetric Higgs mechanism.
} 


\subsection{Vector meson dominance/two site quiver}

Perhaps the simplest and most well motivated model is of vector meson dominance [32-34]. Within this class of model are many variants, depending on the number of vector mesons and also whether the standard model gauge fields couple directly to the hidden sector fields or whether they must couple through a vector meson. We will take as our benchmark the two site quiver model $[9,11,35,36]$, as in figure 1 , as the supersymmetric action is well defined. Considering one vector meson with mass $m_{v}$.

The spectral function may be written to take the form (see also section 5)

$$
\rho=m_{v}^{2} \delta\left(\sigma^{2}-m_{v}^{2}\right)
$$

or equally

$$
\rho=s \delta\left(\sigma^{2}\right)-s \delta\left(\sigma^{2}-m_{v}^{2}\right)
$$

using a manipulation similar to eq. (4.12), resulting in an sfermion mass formula

$$
m_{\phi}^{2}=-(4 \pi \alpha)^{2} \int \frac{d^{4} p}{(2 \pi)^{4}} \frac{1}{p^{2}}\left[\frac{m_{v}^{2}}{p^{2}+m_{v}^{2}}\right]^{2} \Omega\left(\frac{p^{2}}{M^{2}}\right)
$$

after Wick rotation. These models have been explored and generalised [9, 11, 36-40]. Typically one finds three regimes: a) $m_{v} \ll M$ where there are additional contributions to sfermions masses at three loop [9] and the model is "Gaugino Mediated". Conversely in the regime b) $m_{v} \gg M$ the form factor

$$
F\left(p^{2}\right)=\left[\frac{m_{v}^{2}}{p^{2}+m_{v}^{2}}\right]
$$

is $F \simeq 1$. In this case the standard model gauge interactions may be considered to be directly coupled with the supersymmetry breaking sector and not through an intermediate resonance. This will give soft masses of [16]. The hybrid regime c) in which $m_{v} \sim M$ typically has suppressed but non zero scalar soft masses at the high scale and essentially MSSM RG running from the scale $M$ down.

In section 5 we will derive cross sections of $\sigma$ (visible $\rightarrow$ hidden) that reproduce the structure of $\sigma\left(e^{+} e^{-} \rightarrow\right.$ hadrons $)$ as found within the vector meson dominance model.

For a generalised vector meson model the more canonical form of the spectral function is

$$
\rho\left(\sigma^{2}\right)=\sum_{v} \frac{m_{v}^{2}}{g_{v}} \delta\left(\sigma^{2}-m_{v}^{2}\right) .
$$

It is interesting to explore adding to the $\rho(\sigma)=$ resonances + continuum where the continuum piece can take the form of

$$
\rho_{\text {cont. }}=R(s)_{\text {pert. }} \theta\left(\sigma^{2}-s_{c}\right) .
$$

where $R(s)_{\text {pert. }}$ may be chosen to match the perturbative (S)QCD description [41]. Seiberg dual models [42], as they have a single vector meson, are an ideal benchmark construction to test scattering cross sections both at weak and strong coupling in both the electric and dual magnetic descriptions. 


\subsubsection{Old vector meson dominance versus quivers}

The original proposals of vector meson dominance require that the standard model gauge fields interact with hadronic currents via an intermediate vector meson. Generalising this to supersymmetry one may build current vector couplings

$$
\mathcal{L} \supset \int d^{4} \theta\left[g_{S M} \mathcal{J}_{\mathrm{SSM}} \mathcal{V}_{\mathrm{SSM}}+g_{S M} \mathcal{J}_{\rho} \mathcal{V}_{\mathrm{SSM}}+g_{\rho} \mathcal{J}_{\text {SUSY }} \mathcal{V}_{\rho}\right]
$$

and implement an action that couples the standard model gauge field to the vector mesons such as eq. (3.3). Such a model would generate soft masses for the standard model sfermions at the two loop level and would generate a mass for $\lambda_{\rho}$

$$
g_{\rho}^{2} M \tilde{B}_{1 / 2}(0) \lambda_{\rho} \lambda_{\rho}
$$

The standard model gaugino $\lambda_{S M}$ would however remain massless!

Instead if the vector mesons are interpreted as mass eigenstates $\tilde{V}_{i}$ and the currents couple to interaction eigenstates $V_{j}$ with $i=0,1$, as in the quiver models [9, 11, 36-40], the currents couplings are

$$
\mathcal{L} \supset \int d^{4} \theta g_{0} \mathcal{J}_{\mathrm{SSM}} \mathcal{V}_{0}+\int d^{4} \theta g_{1} \mathcal{J}_{\text {SUSY }} \mathcal{V}_{1}
$$

In such a scenario soft masses are generated as in eq. (4.3) for the sfermions and also

$$
g_{S M}^{2} M \tilde{B}_{1 / 2}(0) \tilde{\lambda}_{i} \tilde{\lambda}_{j}
$$

gaugino masses are generated for both $\tilde{\lambda}_{j}$ mass eigenstates. ${ }^{2}$ In addition the quiver model or hidden local symmetry model interprets the vector meson as a gauge boson of an emergent gauge symmetry. It naturally incorporates a gauge invariant (and supersymmetric) Higgs mechanism to generate meson masses.

\subsection{A flat extra dimension}

A well known example is the flat extra dimension $[7,10]$. In these models the Lagrangian is $\mathcal{N}=1$ Super Yang Mills in five dimensions on an $R^{1,3} \times S^{1} / \mathbb{Z}_{2}$ background. The supersymmetric standard model is placed at a fixed point $x_{5}=0$ and a supersymmetry breaking sector located at $x_{5}=\ell$, where $\ell$ is the length of the fifth dimensional interval. In this model the spectral function takes the form

$$
\rho(\sigma)=-\sum_{n} p^{2}(-1)^{n} \delta\left(\sigma^{2}-m_{n}^{2}\right)=\sum_{n} m_{n}^{2}(-1)^{n} \delta\left(\sigma^{2}-m_{n}^{2}\right)
$$

where $m_{n}=n \pi / \ell$. In the above we have used the identity

$$
\delta(0)=\frac{1}{2 \ell} \sum_{n} \frac{p^{2}-m_{n}^{2}}{p^{2}-m_{n}^{2}} .
$$

\footnotetext{
${ }^{2}$ One must also take into consideration the Dirac mass that arises from the action of the quiver.
} 
The current couplings take the form

$$
\mathcal{L} \supset \int d^{4} \theta g \mathcal{J}_{\mathrm{SSM}} \mathcal{V} \delta(y-0)+\int d^{4} \theta g \mathcal{J}_{\text {SUSY }} \mathcal{V} \delta(y-\ell)
$$

where $g_{\mathrm{SSM}}=g_{\text {SUSY }}=g$ : as the interaction eigenstates are mass eigenstates universality of couplings is implemented automatically. The spectral function determines a leading order sfermion soft mass formula

$$
m_{\phi}^{2}=-(4 \pi \alpha)^{2} \sum_{n, n^{\prime}} \int \frac{d^{4} p}{(2 \pi)^{4}} \frac{(-1)^{n+n^{\prime}}}{p^{2}-m_{n}^{2}} \frac{p^{2}}{p^{2}-m_{n^{\prime}}^{2}} \Omega\left(\frac{p^{2}}{M^{2}}\right) .
$$

Completing a Matsubara summation the form factor is given by

$$
F(p \ell)=\left(\frac{p \ell}{\sinh p \ell}\right) \text {. }
$$

The gauge couplings for such a construction are UV sensitive and display a power law running [43]. The key features of the spectral function are that the squared masses scale with $n^{2}$ and that the coefficient oscillates sign, which provides a useful cancellation also for pion form factors [44], a feature that may be modelled with brane to bulk wavefunctions as

$$
f_{n}\left(x_{5}\right) f_{n}\left(y_{5}\right)=\left(e^{i k_{5} \cdot\left(x_{5}-y_{5}\right)}+e^{i k_{5} \cdot\left(x_{5}+y_{5}\right)}\right) .
$$

The oscillating sign has been noted in Large $N_{c}$ form factors also [45]. These models share the same three regimes as the quiver models.

\subsection{General Regge-like Mediation}

Motivated by low energy models of QCD we could assume a Regge-like trajectory [46] which scales with $n$ instead of $n^{2}$

$$
m_{n}^{2}=\mu^{2}(n+S) \text { with } \quad \mu^{2}=\frac{2}{\alpha^{\prime}}
$$

with $\alpha^{\prime}$ associated to the slope of the Regge trajectory, $\mu^{2}$ being the confining string tension. The full Regge trajectory is associated with spin $S$ however we will simply focus on the vector mesons where we take

$$
\rho(\sigma)=\sum_{n} p^{2} F_{n}^{2}(-1)^{n} \delta\left(\sigma^{2}-m_{n}^{2}\right)=-\sum_{n} m_{n}^{2} F_{n}^{2}(-1)^{n} \delta\left(\sigma^{2}-m_{n}^{2}\right) .
$$

We have assumed the $(-1)^{n}$ factor for suitable convergence of the Matsubara summation.

Using the above spectral function will result in

$$
m_{\phi}^{2}=-(4 \pi \alpha)^{2} \sum_{n, n^{\prime}} \int \frac{d^{4} p}{(2 \pi)^{4}} \frac{F_{n}^{2}(-1)^{n+n^{\prime}}}{p^{2}-\mu^{2} n} \frac{F_{n^{\prime}}^{2} p^{2}}{p^{2}-\mu^{2} n^{\prime}} \Omega\left(\frac{p^{2}}{M^{2}}\right) .
$$

For constant $F_{n}=F$ we may complete a Matsubara summation

$$
\sum_{n=-\infty}^{+\infty} \frac{(-1)^{n}}{p^{2}-\mu^{2} n}=\frac{\pi}{\mu^{2}} \operatorname{cosec}\left(\frac{\pi p}{\mu^{2}}\right) .
$$


Using the above identity, unfortunately the relevant momentum integral does not appear to converge: we have in mind a more complete picture [44, 47]. For instance, ideally one would wish to be able to derive a form factor as a ratio of Gamma functions, inspired by the Veneziano amplitude, as was suggested for pions:

$$
F(s) \sim \frac{\Gamma(1-\alpha(s))}{\Gamma(\lambda-\alpha(s))} \frac{\Gamma\left(\lambda-\frac{1}{2}\right)}{\Gamma\left(\frac{1}{2}\right)},
$$

where $2<\lambda<2.5$ fit the pion data quite well [48]. This scales as $F(s) \sim 1 / s^{\lambda-1}$ in the UV. For the case of pions the above result for a $1 \rightarrow 2$ amplitude was obtained from using certain current algebra techniques on a $2 \rightarrow 2$ amplitude and it would be desirable to derive this type of result more directly for this supersymmetric case.

However for $\mu \sim M$ this model will have similar features to that of the two site quiver model (see for instance [40]) with only one resonance. For a minimal messenger sector eq. (5.1), the typical scalar mass result is then

$$
m_{\phi}^{2} \sim\left(\frac{\alpha}{4 \pi}\right)^{2}\left|\frac{F}{M}\right|^{2}\left|\frac{\mu}{M}\right|^{\tau} \quad \text { where } \tau \in[0,2]
$$

with gaugino soft masses unchanged. Surprisingly little has been said about this model in regard to supersymmetry breaking.

\subsubsection{From a soft wall model}

A model that scales with $n$ instead of $n^{2}$ may be constructed [49, 50] using the AdS/CFT perspective. To construct this model one couples $\mathcal{N}=1$ Super Yang Mills in 5 d to a Dilaton multiplet

$$
S=\int d^{5} x \sqrt{g} e^{-\Phi} \mathcal{L}_{S Y M}
$$

whose scalar profile is given by

$$
\Phi(z)=a z^{2}
$$

where $a$ is a positive constant. We choose the Poincaré metric:

$$
d s^{2}=e^{2 A(z)}\left(\eta^{\mu \nu} d x_{\mu} d x_{\nu}-d z^{2}\right) \quad \text { with } \quad A(z)=-\log (z)
$$

such that $G_{M N}=\eta_{M N} / z^{2}$, where $z \in\left[z_{0}, \infty\right)$. In this case we can introduce a supersymmetry breaking sector located at some peak value of $z=L_{1}$ perhaps with a delta function

$$
S \supset \int d^{5} x g^{\prime} \int d^{4} \theta \mathcal{J}_{\text {SUSY }} \mathcal{V}^{\prime} \delta\left(z-L_{1}\right)
$$

or with some smoother profile. This would generate an effective action located around $z=L_{1}$. Exact locality in position space means complete delocalisation in momentum space and it is therefore likely that a smoother profile will dampen couplings to modes with very different Kaluza-Klein number to the incoming mode. 


\subsection{The unparticle limit}

One could imagine an approximately continuous set of resonances [51-53] above some scale $\mu$, which would arise from coupling the standard model to an approximately conformal sector, that mediate the supersymmetry breaking effects.

Let us attempt a crude estimate of the soft terms of such a model. We will not compute the full two loop diagrams but suppose that due to the constraining effects of supersymmetry, ${ }^{3}$ one may use the scalar unparticle two point function on the outer loop for all diagrams: For the ungauge boson, ungaugino and dynamical unD-term, $D_{5} \Sigma$.

The scalar two point function is given by

$$
\begin{aligned}
\Delta\left(p, \mu, d_{s}\right) & =i \frac{A_{d_{s}}}{2 \pi} \int_{\mu^{2}}^{\infty}\left(M^{2}-\mu^{2}\right)^{d_{s}-1} \frac{1}{p^{2}-M^{2}+i \epsilon} d M^{2} \\
& =i \frac{A_{d_{s}}}{2 \sin d_{s} \pi}\left(\mu^{2}-p^{2}-i \epsilon\right)^{d_{s}-2}+\ldots
\end{aligned}
$$

where $[51]$

$$
A_{d_{s}}=\frac{16 \pi^{5 / 2}}{(2 \pi)^{2 d_{s}}} \frac{\Gamma\left(d_{s}+1 / 2\right)}{\Gamma\left(d_{s}-1\right) \Gamma\left(2 d_{s}\right)} .
$$

$d_{s}$ is the scaling dimension. This unparticle description is meant to overlap with that of the holographic models, where in both cases the IR scale or IR brane implement the breaking of conformal symmetry. In the case of an IR cutoff one obtains a mass gap and then a discrete spectrum of massive states. Alternatively a $z$ dependent Dilaton profile coupled to the bulk SYM action [55] may be used to implement a soft breaking of conformality and a continuum spectrum of states.

In the limit that $\mu \ll M$ with a minimal messenger sectior $W=X \Phi \tilde{\Phi}$ the supertracted combination eq. (2.7) of current correlators should be expanded in $p^{2} / M^{2}$ and the leading term is independent of $p[10]$. Therefore we need only evaluate the outer loop

$$
\int \frac{d^{d} y}{(2 \pi)^{d}} \frac{y^{2}}{\left(y^{2}+\Delta\right)^{\alpha}}=\frac{1}{(4 \pi)^{d / 2}} \frac{d}{2} \frac{\Gamma(\alpha-d / 2-1)}{\Gamma(\alpha)} \Delta^{1+d / 2-\alpha}
$$

with $\alpha=4-2 d_{s}$. To couple the ungauge fields to the breaking sector we suggest an operator of the form

$$
S \supset \int d^{4} x \int d^{4} \theta \frac{g^{\prime}}{\Lambda^{d_{s}-1}} \mathcal{J} \mathcal{V}_{\text {unparticle }}^{d_{s}}
$$

where for simplicity $\Lambda=M$. This allows one to repackage $\mu / M$ appropriately and the sfermion masses scale as

$$
m_{\phi}^{2} \sim\left(\frac{\alpha}{4 \pi}\right)^{2}\left(\frac{A_{d_{s}}}{2 \sin d_{s} \pi}\right)^{2} \frac{1}{\Gamma\left(4-2 d_{s}\right)}\left|\frac{F}{M}\right|^{2}\left|\frac{\mu}{M}\right|^{4 d_{s}-2} .
$$

Higher order supersymmetry contributions will certainly effect ungauginos, but as the ungaugino soft mass operator couples to all the ungauginos, it is not entirely clear how resolved individual resonances may become. This is left open for a more systematic study.

\footnotetext{
${ }^{3}$ A book-keeping construction analogous to "theta-warping" may be fruitful here [54].
} 


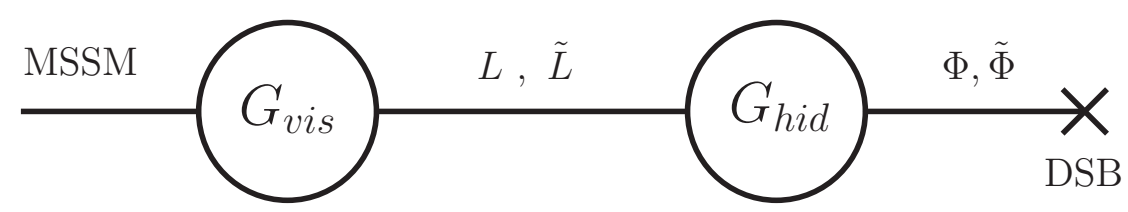

Figure 1. The two site quiver as a toy model.

\section{Scattering: a toy model}

We now turn to scattering which are the main results of this paper. In this section we wish to develop some intuition relating low energy pion and vector meson physics with its SQCD and supersymmetry breaking analogues. Building on this intuition one will be able to extract interesting results for more general situations.

The toy model is the supersymmetric two site quiver $[9,11,36,40,42]$. There is a vector superfield for each gauge group $V_{i}$ where $i=0,1$ labelling the visible and hidden sector respectively. Taking a simple hidden sector superpotential

$$
W=X \Phi \tilde{\Phi}+K\left(L \tilde{L}-v^{2}\right)
$$

The supersymmetry breaking spurion $X=M+\theta^{2} F$ couples to messengers $\Phi, \tilde{\Phi}$. The field $L, \tilde{L}$ are linking fields and the field $K$ is a Lagrange multiplier superfield.

The scalar messenger field mass eigenstates $\phi_{+}, \phi_{-}$have mass squareds $M^{2} \pm F$, the fermions $\psi, \tilde{\psi}$ have mass $M$. It is useful in this analogy to imagine these states as like pions of QCD as they will interact with vector mesons and are ultimately composite, although appear fundamental. Additionally, when we evaluate the correlators their average mass will define the value of the branch cut $s_{0}=4 M^{2}$. The analogy breaks down as the pions are part of an $\mathrm{SU}(2)$ adjoint representation and we are taking the messengers to be in the fundamental.

In the interaction basis there are two $(i=0,1)$ gauge fields $A_{i}^{\mu}$ associated with the visible and hidden sector lattice sites. We will label the external gauge field that couples to the supersymmetric standard model as $A_{0}^{\mu}=\gamma^{\mu}$. In the mass basis we will label $\tilde{A}_{i}^{\mu}$ and in particular the massive state $\rho^{\mu}=\tilde{A}_{1}^{\mu}$, with masses $m_{0}=0$ and $m_{1}=m_{\rho}$. Similarly for the states $\tilde{\lambda}_{1}$ and $\tilde{\lambda}_{2}^{\alpha}=\rho^{\alpha}$.

In these two site models

$$
\frac{1}{g_{S M}^{2}}=\frac{1}{g_{0}^{2}}+\frac{1}{g_{1}^{2}} \quad g_{S M}^{2}=\frac{g_{0}^{2} g_{1}^{2}}{g_{0}^{2}+g_{1}^{2}}
$$

where

$$
m_{v}^{2}=4 v^{2}\left(g_{0}^{2}+g_{1}^{2}\right)=4 v^{2} \frac{g_{0}^{2} g_{1}^{2}}{g_{S M}^{2}} .
$$

One can identify the mass eigenstates

$$
\rho_{\mu}=\frac{g_{0} \gamma_{\mu}-g_{1} A_{\mu}^{1}}{\sqrt{g_{0}^{2}+g_{1}^{2}}} \text { and } A_{\mu}=\frac{g_{1} \gamma_{\mu}+g_{0} A_{\mu}^{1}}{\sqrt{g_{0}^{2}+g_{1}^{2}}} .
$$


In the scattering process of $\gamma^{\mu}$ to $\phi_{+}, \phi_{-}$there is an intermediate $\rho^{\mu}$ as a mass eigenstate. Crucially, the form factor for $A \rightarrow B+\gamma$ for A, B being hidden sector fields, may be related to the form factor of the process $A \rightarrow B+A_{1}^{\mu}$

$$
F_{A B}^{\gamma}\left(q^{2}\right)=\left[\frac{m_{\rho}^{2}}{m_{\rho}^{2}+q^{2}}\right] F_{A B}^{A_{\mu}^{1}}\left(q^{2}\right)=-\sum_{n=0}^{1} \frac{(-1)^{n}}{q^{2}+m_{n}^{2}} q^{2} F_{A B}^{A_{\mu}^{1}}\left(q^{2}\right)
$$

where on the right hand side we have used the "bulk propagator" which is derived from the overlap of the interaction basis with the mass eigenstates [56]. One is therefore able to define a "modified current operator" [23]

$$
\left\langle B\left|\hat{J}^{\gamma, \mu}\right| A\right\rangle=D^{\mu \nu}\left(q^{2}\right) q^{2}\left\langle B\left|J_{\nu}^{A_{\nu}^{1}}\right| A\right\rangle
$$

where a given amplitude $\mathcal{M}$ is found from the Heisenberg field $j^{\mu}$,

$$
\mathcal{M}^{\mu}(p)=\int d^{4} x e^{i p \cdot x}\left\langle B\left|j^{\mu}(x)\right| A\right\rangle .
$$

One takes the absolute square of this matrix element to compute cross sections. Such an identity is crucial in the vector meson dominance program in $e^{+}, e^{-} \rightarrow$ hadrons and similarly for $e^{+} e^{-} \rightarrow \mu^{+} \mu^{-}$with intermediate $\phi(1020)$ and also $\omega(782)$ decays [34]. Here we predict the same feature in $e^{+} e^{-} \rightarrow$ hidden.

It would therefore be possible to measure $g_{0}$ and the combination $g_{S M}^{2}$ independently. This is done by measuring ${ }^{4}$

$$
\frac{g_{0}^{2}}{4 g_{S M}^{2}}=\frac{1}{g_{0}^{2}} \int_{s_{0}} d s^{\prime} \sigma_{a}\left(\text { visible } \rightarrow \rho \rightarrow \phi_{+}, \phi_{-} s^{\prime}\right)
$$

where the cross section $\sigma$ is related to the current correlators $\tilde{C}_{a}(s)$ [13]. Measurements of this type would be a direct test of the magnetic gauge coupling $g_{1}$ in Seiberg dual models of the form [42, 57]. It is also an example of an integral of a cross section. Let us now demonstrate a sum rule from another intergral over a cross section.

\subsection{A sum rule}

It is interesting to also explore possible sum rules that may arise. For the case of a single vector meson as in the above model, it is possible to construct a sum rule for the cross sections that will apply for all $\tilde{C}_{a}(s)$ separately. Defining

$$
\Sigma_{\rho \rho}^{v i s}\left(m_{\rho}^{2}\right)=m_{\rho} \Gamma(\rho \rightarrow v i s) \text { and } \Sigma_{\rho \rho, a}^{h i d}\left(m_{\rho}^{2}\right)=m_{\rho} \Gamma_{a}(\rho \rightarrow h i d)
$$

$\left(\Sigma_{\rho \rho}^{v i s}\left(m_{\rho}^{2}\right)\right.$ carries a factor $\left.4 \pi \alpha^{2}\right)$ where in general one has

$$
\Sigma_{V_{1} V_{2}}^{h}=\frac{1}{6} S_{F}(2 \pi)^{4} \delta_{a}\left(p-p_{F}\right)\left\langle h\left|J_{\mu}^{V_{1}}(0)\right| 0\right\rangle\left\langle h\left|J_{\nu}^{V_{2}}(0)\right| 0\right\rangle^{*} \eta^{\mu \nu}
$$

\footnotetext{
${ }^{4}$ This may take different forms depending on where one interprets $g_{0}=g_{S M}$ or as in eq. (5.2).
} 
where $\langle h|$ labels hidden sector states and $S_{F}$ being the standard phase space integration. Redefining the form factor of the modified current operator to be

$$
F(s)=\left[\frac{-g_{e} m_{\rho}^{2}}{g_{\rho}} \frac{g_{\rho \phi \tilde{\phi}}}{s-D(s)}\right]
$$

one can write

$$
\sigma_{a}(v i s \rightarrow h i d)=\frac{12 \pi}{s} \frac{\sum_{\rho \rho}^{v i s}(s) \sum_{\rho \rho, a}^{h i d}(s)}{|s-D(s)|^{2}}
$$

where $D(s)=m_{\rho}^{2}+\Pi(s)$, the last term being the self energy. Using the dispersion relation

$$
\frac{1}{D(s)-s}=\frac{1}{\pi} \int_{s_{0}}^{\infty} \frac{\operatorname{Im} D\left(s^{\prime}\right)}{\left|D\left(s^{\prime}\right)-s^{\prime}\right|^{2}} \frac{d s^{\prime}}{s^{\prime}-s+i \epsilon}
$$

with $D\left(m_{\rho}\right)=m_{\rho}^{2}-i m_{\rho} \Gamma$, the sum rule is

$$
\frac{m_{\rho}^{2}}{D_{b}(0)}=\frac{g_{\rho \phi \tilde{\phi}}}{g_{\rho}}=\frac{1}{12 \pi^{2} m_{\rho}} \sum_{\text {cuts }} \int_{s_{0}}^{\infty} s \sigma_{b}(v i s \rightarrow \rho, s) d s / \Gamma(\rho \rightarrow v i s)=x_{b, \rho}
$$

This is a correction parameter due to the finite width resonance. In the zero width approximation $D(0)=m_{\rho}^{2}$ which leads to $g_{\rho \phi \tilde{\phi}}=g_{\rho}$ in eq. (3.4). Taking $D(0)=a_{\rho} m_{\rho}^{2}$ at finite width and measuring $x_{\rho}$ in conjunction with $m_{\rho}$ can be used to measure the value of $a$ in $[42,57]$. If $x_{b, \rho}=x_{\rho}$ for all correlators, implying a new supersymmetric type of universality of form factors, this would be a highly nontrivial check on the emergence of a hidden local symmetry. It can be shown that the sum of $\sum_{v} x_{v} m_{v}^{2} / g_{v}=C_{S c h}$ defines the Schwinger term in equal time commutators. There may be further sum rules based on already known QCD sum rules such as this.

It is also natural to define the messenger's effective charge radius

$$
\left\langle r^{2}\right\rangle=-\left.6 \frac{\partial}{\partial s} F(s)\right|_{s=0}
$$

which for the two site model above is given by

$$
\left\langle r^{2}\right\rangle=\frac{6}{m_{\rho}^{2}}
$$

In summary, for this simple two site quiver the variables $g_{1}, m_{\rho}$ and $x_{\rho}$ (and therefore $a_{\rho}$ ) may all be determined from measuring scattering cross sections of visible $\rightarrow$ hidden sector states, which is directly related to the proposals of $[42,57]$.

\section{$6 \quad$ Scattering cross sections}

In [20] cross sections $^{5}$ from hidden sector current correlators were computed. This section explores the effect of intermediate states in these cross sections of visible+visible $\rightarrow$ hidden processes. It is hoped that these results will give some insight into connecting the theoretical 


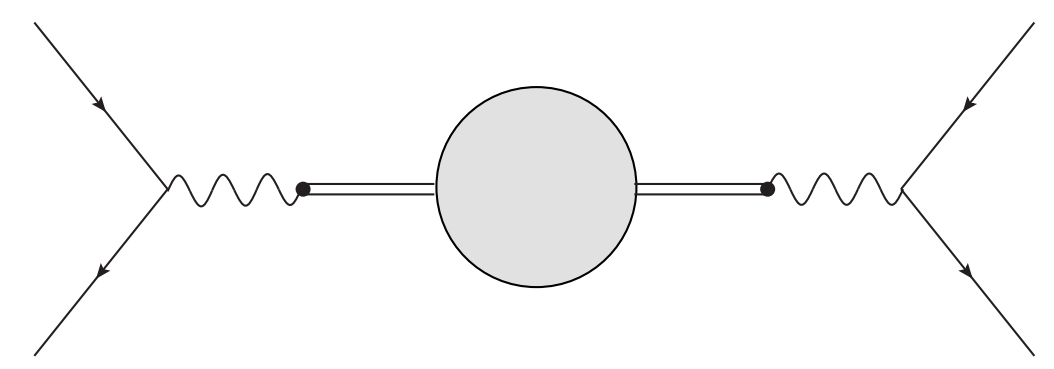

Figure 2. A diagram to represent the matrix element $\mathcal{M}\left(e^{+} e^{-} \rightarrow e^{+} e^{-}\right)$for which one applies the optical theorem to obtain $\sigma\left(e^{+} e^{-} \rightarrow\right.$ hidden $)$. The double lines denote an intermediate vector resonance or resonances, depending on the model. The blob denotes the current correlator $\tilde{C}_{1}^{\prime}(s)$. The cut is applied across the correlator.

models with observation. In particular the approach taken here is form factor based and it would be interesting to develop methods to overlap this with the OPE approach of [20].

In general gauge mediation, observable cross sections, for energies large enough to create on shell hidden sector states, may be extracted from the current correlators by application of the optical theorem:

$$
\sigma_{a}(\text { vis } \rightarrow \text { hidden })=\frac{(4 \pi \alpha)^{2}}{s} \frac{1}{2} \text { Disc } \tilde{C}_{a}(s) .
$$

where $s$ is the Mandelstam variable, center of mass energy squared associated with the discontinuity across the cut. Conversely, in principle one could completely determine the current correlators from the experimental cross sections:

$$
i\left(16 \pi^{2} \alpha\right)^{2}\left[\tilde{C}_{a}(s)-\tilde{C}_{a}(0)\right]=\frac{s}{\pi} \int_{s_{0}}^{\infty} d s^{\prime} \frac{\sigma_{a}\left(s^{\prime}\right)}{s^{\prime}-s},
$$

where $s_{0}$ is the end point of the cut and a sum over multiple cross sections is implicit. Hence, there is an important relationship between sfermion masses and in-principle observable cross sections. If one were to assume, as in QCD, intermediate resonances $\sigma$ (vis $\rightarrow V_{n} \rightarrow$ hidden), then this vector meson saturated form factor may be pulled outside of the current matrix element and hence outside of the current correlator. In other words, and to make clear the correspondence with generalised vector meson dominance and current matrix elements, we should associate

$$
\tilde{C}_{1}(s) \Pi_{\mu \nu} \sim\left\langle h\left|J_{\mu}^{\mathrm{em}}\right| 0\right\rangle\left\langle h\left|J_{\nu}^{\mathrm{em}}\right| 0\right\rangle^{*}
$$

where $h$ labels the hidden sector fields and the matrix element couples to the external electromagnetic field. The defining relationship between matrix elements is given by

$$
\left\langle B\left|J_{\mu}^{\mathrm{em}}\right| A\right\rangle=\int d \sigma^{2} \frac{\rho\left(\sigma^{2}\right)}{s-\sigma^{2}+i \sigma \Gamma}\left\langle B\left|J_{\mu}^{\rho_{n}}\right| A\right\rangle=F(s)\left\langle B\left|J_{\mu}^{\rho_{n}}\right| A\right\rangle .
$$

where we label $F(s)$ the form factor to simplify notation. For scattering, the spectral function to use is $\rho(s)=m_{n}^{2} \ldots$, for example as in eq. (4.11).

\footnotetext{
${ }^{5}$ See also pages 242 e.q. (5-155a) and page 310 of [58].
} 
As a result, for a general resonance model, the contribution from intermediate s-channel resonances may be written

$$
\sigma_{a}(\text { vis } \rightarrow \text { hidden })=\frac{(4 \pi \alpha)^{2}}{2 s} \int d \sigma^{2} \frac{\rho\left(\sigma^{2}\right)}{s-\sigma^{2}+i \sigma \Gamma} \int d \sigma^{\prime 2} \frac{\rho\left(\sigma^{\prime 2}\right)}{s-\sigma^{\prime 2}-i \sigma \Gamma} \operatorname{Disc} \tilde{C}_{a}^{\prime}(s) .
$$

With $\Gamma$ the decay width. The $\tilde{C}_{a}^{\prime}(s)$ with a ', now being the correlator of current matrix elements relating vector mesons to hidden sector fields:

$$
\tilde{C}_{1}^{\prime}(s) \Pi_{\mu \nu} \sim\left\langle h\left|J_{\mu}^{\rho_{n}}\right| 0\right\rangle\left\langle h\left|J_{\nu}^{\rho_{m}}\right| 0\right\rangle^{*} .
$$

This is pictured in figure 2 .

Finally, to see that this is the correct result we display the canonical spectral function eq. (4.5)

$$
\rho(\sigma)=\sum_{v} \frac{m_{v}^{2}}{g_{v}} \delta\left(\sigma^{2}-m_{v}^{2}\right),
$$

completely reproducing known results for generalised vector meson dominance. In this way one may obtain a cross section for these resonance models from the cross sections of [20]. The above result is obtained in an entirely four dimensional construction by assuming a generalised form of vector meson dominance. However, a holographic interpretation can be made in which the form factors are associated with a bulk to boundary five dimensional propagator. Finally applying this understanding of cross sections in reverse, one can then infer the form of the sfermion mass formula to be eq. (2.13).

To clarify this little further, one interprets the correlators of [20] to be associated with vector meson-hidden interactions and assume that the hidden sector has some weakly coupled description such as minimal gauge mediation with superpotential $W=X \Phi \tilde{\Phi}$. To compute the correlator ones starts with the cross section associated with the scalar current

$$
J^{a}=\phi^{\dagger} T^{a} \phi-\tilde{\phi} T^{a} \tilde{\phi}^{\dagger}=\phi_{+}^{\dagger} T^{a} \phi_{-}+\phi_{-}^{\dagger} T^{a} \phi_{+} .
$$

This will produce two hidden sector scalars of masses $m_{+}$and $m_{-}$,

$$
\sigma_{0}(s)=(4 \pi \alpha)^{2}|F(s)|^{2} \frac{\lambda^{1 / 2}\left(s, m_{+}, m_{-}\right)}{8 \pi s^{2}}\left|\frac{\mathcal{M}}{4 \pi \alpha}\right|^{2}
$$

$\mathcal{M}$ is the corresponding matrix element for this process. ${ }^{6}$ The phase space or 'triangle factor' is given by

$$
\lambda^{1 / 2}\left(s, m_{1}, m_{2}\right)=2 \sqrt{s}|\vec{p}|=\sqrt{\left[s-\left(m_{1}+m_{2}\right)^{2}\right]\left[s-\left(m_{1}-m_{2}\right)^{2}\right]} \theta\left(s-\left(m_{1}+m_{2}\right)^{2}\right)
$$

$\theta$ is the unit step function, for positive values of its argument. One can use the optical theorem to obtain ${ }^{7}$

$$
\frac{1}{2} \operatorname{Disc} \tilde{C}_{0}^{\prime}(s)=\frac{1}{2}\left(\tilde{C}_{0}^{\prime}(s+i \epsilon)-\tilde{C}_{0}^{\prime}(s-i \epsilon)\right)=\frac{1}{4 \pi s} \sqrt{s^{2}-4|M|^{2}+4|F|^{2}} .
$$

\footnotetext{
${ }^{6}$ Note that we have defined dimensionless spectral functions which account for a factor of $s$.

${ }^{7}$ See page 310 of [58].
} 
The final cross section is given by

$$
\sigma_{0}(\text { vis } \rightarrow \text { hidden, } s)=(4 \pi \alpha)^{2}|F(s)|^{2} \frac{1}{4 \pi s^{2}} \sqrt{s^{2}-4|M|^{2}+4|F|^{2}} .
$$

There may be additional kinematic factors for the other cross sections associated with $\tilde{C}_{a}^{\prime}(s)$ for $a=0,1 / 2,1$. Also, in the two site model for instance, these results are modified to take account of $g_{0}, g_{1}$ gauge couplings at each lattice site. It is straightforward to extend the results of [20] in this way. It is notable that for the resonance masses $m_{n}^{2}(1+2 n)$, the cross sections can acquire an approximately scale invariant form $[19,59]$.

Let us now emphasise the close relationship between these cross sections and the spinless external states of hadrons in QCD, for definiteness we focus on $\sigma\left(e^{+}, e^{-} \rightarrow \pi_{+}, \pi_{-}\right)$. The pions of QCD are in the adjoint of SU(2) where the current is $J_{\pi}^{\mu}=\vec{\pi} \times \partial^{\mu} \vec{\pi}$ whereas in this case the messengers are taken to be in the fundamental in this example. This is meant as an instructive analogy. To do this take $F \rightarrow 0$ and identify $M_{S U S Y}$ with $M_{\pi}$. In this case the phase space factor reduces to the simple form

$$
\lambda^{1 / 2}(s, m, m)=\left[s\left(s-4 m^{2}\right)\right]^{1 / 2}=s \sqrt{1-\frac{4 m^{2}}{s}} \theta\left(s-4 m_{\pi}^{2}\right) .
$$

The actual correlator of the pion current is

$$
\tilde{C}_{\pi}(s)=\frac{2}{3 p^{2}} \int \frac{d^{4} q}{(2 \pi)^{4}} \frac{(p+k)_{\mu} k^{\mu}}{(p+k)^{2}+m_{\pi}^{2}} \frac{1}{k^{2}+m_{\pi}^{2}}
$$

giving

$$
\operatorname{Disc} \tilde{C}_{\pi}\left(p^{2}\right)=\frac{1}{12 s^{2}}\left(s-4 m_{\pi}^{2}\right) \lambda^{1 / 2}\left(s, m_{\pi}, m_{\pi}\right)
$$

and therefore

$$
\sigma\left(e^{+}, e^{-} \rightarrow \pi^{+}, \pi^{-}, s\right)=\frac{(4 \pi \alpha)^{2}}{4 \pi 12 s}\left(1-\frac{4 m^{2}}{s}\right)^{3 / 2}|F(s)|^{2} \theta\left(s-4 m^{2}\right) .
$$

One can straightforwardly extend this approach to many other cross sections, including higher spin states, and for instance if one assumes supersymmetry breaking sector baryons $B_{h}$, then using eq. (6.4) the typical scattering of off-shell standard model visible gauge fields $\gamma$ or $\lambda$ to $B_{h}$ will essentially take the form

$$
\sigma_{\gamma^{*} B_{h}}^{T}(s)=(4 \pi \alpha)|F(s)|^{2} \sigma_{\rho_{n} B_{h}}^{T}(s)
$$

where typically a multiplying factor $s / m_{n}^{2}$ is necessary for off-shell longitudinal gauge fields.

That this construction reproduces already known results for vector meson dominance cross sections is a powerful confirmation of this approach. Furthermore, whilst the results may be motivated by " $5 \mathrm{~d}$ " bulk to boundary propagators, the result may be thought of as entirely four dimensional. This is simply a direct application of the "modified current operator". It is also easy to calculate and simple to fit to (future) data. Whilst these cross sections are associated with some scale $M$, typically hundreds of $\mathrm{TeV}$ and therefore out of reach of present accelerators, it is hoped these results give some important insights for model building of supersymmetry breaking. 
In [20] the operator product expansion (OPE) was used to determine the sfermion masses of [16]. Essentially the OPE is a $1 / s=M^{2} / p^{2}$ expansion. For models where resonances contribute to the mediation, the dominant contribution to the integral for sfermion masses is for small $s$, where an $p^{2} / M^{2}$ expansion is more appropriate. The OPE expansion still correctly determines the sfermion soft masses in the limit $m_{\rho} \gg M$, although unfortunately this is not the region of interest.

\section{The A-term}

In this section we wish to show that the feature of the spectral function also arises for Aterms. The leading gauge mediated A-term contribution is the third generation $a_{t} Q \bar{u} H_{u}$. In resonance mediation, with no additional features, it is given by

$$
a_{t}=-\frac{8 y_{t}}{3}\left(4 \pi \alpha_{3}\right)^{2} \int \frac{d^{4} p}{(2 \pi)^{4}} \int d^{2} \sigma \frac{\rho\left(\sigma^{2}\right)}{s-\sigma^{2}} \int d^{2} \sigma^{\prime} \frac{\rho\left(\sigma^{\prime 2}\right)}{s-\sigma^{\prime 2}} \frac{M B_{1 / 2}\left(p^{2} / M^{2}\right)}{\left(p^{2}-m_{t}^{2}\right)^{2}}
$$

As a two loop diagram it is severely suppressed if the sfermions mass formula is suppressed, at the high scale $M$, by the same form factor as the sfermion masses. For the case of a simple two site quiver and minimal gauge mediation sector, it may be evaluated exactly using identities from [60, 61].

In the $4 \mathrm{~d}$ limit in which the resonance scale is much heavier than $M$, and with the minimal model $W=X \Phi \tilde{\Phi}$ behaves as

$$
A_{t}(4 d)=-y_{t} \frac{8}{3}\left(\frac{\alpha_{3}}{4 \pi}\right)^{2} m_{0}(h(a, b)-h(a, c))
$$

where $h(a, b)$ is a function given in $[60,61]$. The function $h$ is given by the integral

$$
h(a, b)=\int_{0}^{1} d x\left(1+\operatorname{Li}_{2}\left(1-\mu^{2}\right)-\frac{\mu^{2}}{1-\mu^{2}} \log \mu^{2}\right)
$$

The dilogarithm is defined as $\operatorname{Li}_{2}(x)=-\int_{0}^{1} \frac{d t}{t} \log (1-x t)$ with

$$
\mu^{2}=\frac{a x+b(1-x)}{x(1-x)} \quad, \quad a=m_{1}^{2} / m_{0}^{2}, \quad b=m_{2}^{2} / m_{0}^{2}
$$

and

$$
a=\frac{m_{0}^{2}}{m_{t}^{2}}, \quad b=\frac{m_{+}^{2}}{m_{t}^{2}} \quad c=\frac{m_{-}^{2}}{m_{t}^{2}}
$$

Taking the leading log pieces with $y=M / m_{t}$ and $x=F / M^{2}$

$$
(h(a, b)-h(a, c))=(\log [1-x]-\log [1+x]) \log [y]+\ldots
$$

In the limit of massless $m_{t}$ it behaves as

$$
(h(a, b)-h(a, c)) \sim-x,
$$


which vanishes as $F \rightarrow 0$. The $A_{t}$ is a two loop effect, which is further suppressed by the presence of resonances. A viable model may therefore require further extensions to overcome this issue, many have already been proposed which involve direct couplings between Higgs multiplets and the supersymmetry breaking $[1,62]$ or by modifying $\mathrm{D}$ terms using vector mesons [37]. This result is of course well known but it is included it to highlight the appearance of the form factors, which are in principle observable from cross sections, in various loops.

\section{Holography and the generating functional}

In this section we wish to apply a holographic model as an effective theory of a strongly coupled large $N_{c}$ SQCD sector that breaks supersymmetry. We assume that this $\mathrm{SU}\left(N_{c}\right)$ has in addition some global symmetry $\mathrm{SU}\left(N_{F}\right)$ which is weakly gauged and associate with the standard model gauge groups. This demonstrates much of the utility of general gauge mediation [3] and is a concrete model from which to extract the various form factors for both sfermion masses and cross sections. This section overlaps with the hidden local symmetry program of AdS/QCD [63] and shares features with $[8,64]$, which we will abbreviate to AdS/SUSY.

In analogy to hidden local symmetry models [63], we apply a type of Wilsonian matching, where we wish to analyse a system which is described by an effective theory

$$
Z_{\mathrm{SSM}}[\mathbb{J}] \times\left. Z_{S Q C D}[\mathcal{J}]\right|_{E \ll \Lambda} \rightarrow Z_{\mathrm{SSM}}[\mathbb{J}] \times Z_{\mathrm{AdS}}[\mathcal{O}] \times Z_{\mathrm{IR}}[J] .
$$

The effective action is the bare action or the classical action at the scale $\Lambda$. The theory on the left hand side is four dimensional. The effective description on the right hand side is five dimensional in which we have introduced an additional dimension $z$. We make the assumption that we can match the observable physics on both sides for energies around the matching scale $\Lambda$. Then at energies $E \ll \Lambda$ we wish to use the generating functional on the right hand side to compute physical quantities. In particular this means that instead of attempting to solve certain equations of motion with current sources on the IR brane, we can use perturbation theory.

The generating functional for the supersymmetric standard model we will label by

$$
Z_{\mathrm{SSM}}[\mathbb{J}]=\int D\left[S S M, V(x)=\tilde{V}\left(x, z=L_{0}\right)\right] e^{i S(S S M, V(x) ; \mathbb{I})}
$$

Where we take the $S S M=Q U D L E H_{u} H_{d}$, the supersymmetric standard model fields, which we locate on the UV boundary (outside the AdS system).

The large $N_{c}$ strongly coupled supersymmetry breaking sector we will label SQCD:

$$
Z_{S Q C D}=\int D[Q, \tilde{Q}, G] e^{i S(Q, \tilde{Q}, G ; \mathcal{J})}
$$

We assume that at energies $E \ll \Lambda$ it admits a dual "slice of AdS"

$$
Z_{\mathrm{AdS}}[\mathcal{O}]=\int D\left[\tilde{V}, \Phi_{A d j}\right] e^{i S\left(\tilde{V}, \Phi_{A d j} ; \mathcal{O}\right)}
$$


The $\tilde{V}+\Phi_{A d j}$ are the superfields of $\mathcal{N}=1 \mathrm{SYM}$ in $5 \mathrm{~d}$ [65]. We take the supergravity background to be static but those degrees of freedom should really be included in an analysis of hidden $\rightarrow$ hidden scattering, for example. For our purposes we are interested in modeling the behaviour of the gauge field in this background. Importantly, we have chosen the $\tilde{V}$ to have positive parity (and therefore a massless zero mode spectrum) and $\Phi$ to have negative parity and no massless modes. This choice is consistent with phenomenology. The supersymmetric action plus parity completely determines the field profiles and the relative field profiles between each field in the same multiplet.

In the dual description we expect that the actual supersymmetry breaking is located on the IR brane

$$
Z_{\mathrm{IR}}[J]=\int D\left[X, \Phi, \tilde{\Phi}, V_{\mathrm{IR}}=\tilde{V}\left(x, z=L_{1}\right)\right] e^{i S\left(\Phi, \tilde{\Phi}, V_{\mathrm{IR}} ; J\right)}
$$

The field $X$ is the supersymmetry breaking spurion, $\Phi, \tilde{\Phi}$ are the messenger fields, that are coupled directly to the spurion $X$ and from which the global currents of $J$ are extracted. In principle one may locate the supersymmetry breaking fields in the bulk and remove the IR brane. We choose the above construction for visual clarity. The hidden sector is therefore the combination of $Z_{\mathrm{AdS}}[\mathcal{O}] \times Z_{\mathrm{IR}}[J]$ and in particular the resonances of $\tilde{V}+\Phi_{\text {Adj }}$ are composites of the hidden sector with standard model quantum numbers.

In this model both the UV and IR brane fields are dynamical and must be integrated over. At each point along the AdS direction is the field $\tilde{V}(x, z)$ and specifically its UV limit $V$ is the external gauge field that couples to the supersymmetric standard model fields. At the IR brane, the supersymmetry breaking effects are encoded in $J$ and couple to $V\left(z=L_{1}\right)$ [66-69].

In terms of the $A d S_{5}$ direction $z$, this corresponds to $z=L_{1}$. In the first instance the supersymmetry breaking effects, which are encoded in the currents labelled $J$, do not couple directly to $A_{\mu}\left(x, z=L_{0}\right)$ but instead to $A_{\mu}\left(x, z=L_{1}\right)$, a characteristic of locality in theory space. Furthermore, as $\alpha_{S M} \rightarrow 0$ all effects, such as soft masses, of supersymmetry being broken must vanish.

In particular we are not breaking supersymmetry using boundary conditions. Instead we have a dynamical sector located on the IR brane which spontaneously breaks supersymmetry and for which we can clearly define a goldstino field. In addition this will allow us to compute cross sections to messenger fields. We also make the requirement that our messenger sector satisfies the sum rule $\operatorname{Str} \mathcal{M}^{2}=0$ before gravity effects are taken into account.

\subsection{Holographic gauge fields}

In this section we will define the relevant parts of the gauge theory living inside the $Z_{\mathrm{AdS}}[\mathcal{O}]$. We will take $\mathcal{N}=15 \mathrm{D}$ Super Yang Mills on a slice of $A d S_{5}$ background $[54,65]$ with coupling $g_{5}$. We will then couple the supersymmetry breaking sector located at $Z_{\mathrm{IR}}[\mathrm{J}]$.

Starting with the conformally flat metric with mostly minus signature

$$
d s^{2}=a^{2}(z)\left(\eta^{\mu \nu} d x_{\mu} d x_{\nu}-d z^{2}\right)
$$


where

$$
a=\left(\frac{R}{z}\right)=\left(\frac{1}{\omega z}\right)
$$

$R \equiv L$ is the AdS curvature radius, with $L_{0}$ the position of the UV brane and $L_{1}$ the position of the IR brane. We may relate $R^{4}=4 \pi l_{s}^{4} g_{s} N_{c}$, where the string coupling is related $g_{s}=g_{Y M}^{2}$ to the boundary $\mathrm{SU}\left(N_{c}\right)$ gauge coupling $g_{Y M} \cdot l_{s}^{2}=\alpha^{\prime}$ is the string length. The 't Hooft coupling is $\lambda_{t}=g_{Y M}^{2} N_{c}$. See also [70].

One may interchange between conformal coordinates and warped coordinates of "proper distance" using

$$
\frac{z}{R}=e^{k y} \quad \partial_{y}=a^{-1} \partial_{z} \quad d y=a(z) d z \quad \sigma=\ln \left(\frac{z}{R}\right)
$$

where $k$ is the AdS warp factor. The kinetic terms for the super Yang Mills action is given by

$$
\int d^{5} x \operatorname{tr}\left[-\frac{1}{2} F_{\mu \nu} F^{\mu \nu}(z)+i\left(\frac{R}{z}\right)^{3} \bar{\lambda} \sigma^{\mu} \partial_{\mu} \lambda(z)+\frac{1}{2}\left(\frac{R}{z}\right)^{4} D^{2}(z)\right] .
$$

The bulk vector super fields have a Kaluza-Klein decomposition [66, 71]

$$
V(x, z)=\sum_{n} V_{n}(x) f_{n}^{(2)}(z) \text { and } \Phi(x, z)=\sum_{n} \Phi_{n}(x) g_{n}^{(4)}(z) .
$$

The vector superfield and chiral superfield having even and odd parity, respectively. Once an IR and UV brane is implemented the bulk fields will obtain a KK spectrum. As a result the parities of the bulk fields have been chosen so that massless modes such as $\chi \subset \Phi$ do not make the spectrum of the model phenomenologically incompatible. If this field were given even parity and a bulk $\mathcal{N}=2$ SYM with 8 supercharges, then a soft Dirac mass could in principle be generated between $\chi$ and $\lambda$, but one may require exact $\langle\Phi\rangle_{0}^{a} T^{a} \equiv 0$. We have chosen $\Phi$ to have odd parity and so no Dirac mass may arise.

The solutions of the KK wave functions are given by

$$
\begin{aligned}
& f_{n}^{s}(z)=\frac{1}{N_{n}} a^{(s / 2)}\left[J_{1}\left(m_{n} z\right)+b_{\alpha}\left(m_{n}\right) Y_{1}\left(m_{n} z\right)\right] \\
& g_{n}^{s}(z)=\frac{\epsilon(z)}{N_{n}} a^{(s / 2)}\left[J_{0}\left(m_{n} z\right)+b_{\alpha}\left(m_{n}\right) Y_{0}\left(m_{n} z\right)\right]
\end{aligned}
$$

where $\epsilon(z)$ is a sign function. The positive parity boundary conditions on the branes fix

$$
b\left(m_{n}\right)=-\frac{J_{1}\left(m_{n} R\right)}{Y_{1}\left(m_{n} R\right)} .
$$

The orthonormality condition is given by

$$
\int a^{(s-2)} f^{s}(z) f^{s}(z) d z=\delta_{n m}
$$

It is often useful to use

$$
\partial_{z} g^{(4)} \partial_{z} g^{(4)} \simeq m_{n}^{2} f^{(2)} f^{(2)} .
$$

One can then define a bulk AdS two point function for the vector fields,

$$
G\left(p^{2} ; z, z^{\prime}\right)=\sum_{n} \frac{f_{n}^{(2)}(z) f_{n}^{(2)}\left(z^{\prime}\right)}{p^{2}-m_{n}^{2}}
$$




\subsection{Soft masses and cross sections}

Suppose one now integrates out $Z_{\mathrm{IR}}[J]$ (one may later integrate out $Z_{\mathrm{AdS}}[\mathcal{O}]$ ). Introducing a specially normalised source term

$$
g_{5} \int d^{5} x \delta\left(z-L_{1}\right)\left(a^{2} \hat{J} D-a^{3 / 2} \hat{j}^{\alpha} \lambda_{\alpha}-a^{3 / 2} \hat{\bar{j}}_{\dot{\alpha}} \bar{\lambda}^{\dot{\alpha}}-\hat{j}^{\mu} A_{\mu}\right)
$$

where $a=R / z$. The presence of currents will change the equations of motion to include source terms. Integrating out this sector, this will generate an effective action for the gauge supermultiplet that couples to $V\left(x, z=L_{1}\right)$ :

$$
\begin{aligned}
\mathcal{L}_{\text {eff }}= & \frac{g_{5 d}^{2}}{2} \tilde{C}_{0}(0) D^{2}(z)\left(\frac{R}{z}\right)^{4}-g_{5 d}^{2} \tilde{C}_{1 / 2}(0) i \lambda \sigma^{\mu} \partial_{\mu} \bar{\lambda}(z)\left(\frac{R}{z}\right)^{3}-\frac{g_{5 d}^{2}}{4} \tilde{C}_{1}(0) F_{\mu \nu} F^{\mu \nu}(z) \\
& -\frac{1}{2} g_{5 d}^{2} M \tilde{B}_{1 / 2}(0)(\lambda \lambda(z)+\bar{\lambda} \bar{\lambda}(z))\left(\frac{R}{z}\right)^{3}+\ldots
\end{aligned}
$$

the ellipses denote higher order terms in $g_{5 d}^{2}$ and higher orders in momentum, and we take $z=L_{1}$. The first line of eq. (8.18) corresponds to wavefunction renormalisation caused by integrating out the fields $X, \Phi, \tilde{\Phi}$, and will in turn cause a change in the beta function $\Delta b_{\mathrm{IR}}$ but this will not have much effect above the scale $E \sim 1 / L_{1}$. Canonically normalised currents have been used such that the mass scales are given by

$$
\left(\frac{R}{z}\right)^{2} F=\hat{F} \quad\left(\frac{R}{z}\right) M=\hat{M}
$$

In this way one may compute the loops of messenger fields. The masses of the messenger fields are now

$$
m_{ \pm}^{2}=\hat{M}^{2} \pm \hat{F} \text { and } m_{0}^{2}=\hat{M}^{2} .
$$

One then uses this effective action to generate soft masses for the SSM located at $z=L_{0}$.

\subsubsection{Soft masses in general}

The gaugino soft mass is given, after canonically normalising the kinetic term of $\lambda$ in eq. (8.9) relative to the soft mass of eq. (8.18), by

$$
m_{\lambda n, m}=(4 \pi \alpha) \hat{M} \tilde{B}_{1 / 2}(0) f_{n} f_{m}+\text { c.c. }
$$

as it is at $p_{\text {ext }}=0$ it is unnaffected by form factors: the physical gaugino mass is the shift pole in the propagator, whilst there are form factors in cross sections to $\sigma_{1 / 2}^{\prime} \sim$ $G\left(z, z^{\prime}\right) G\left(z, z^{\prime}\right) \operatorname{Disc} \hat{M} \tilde{B}_{1 / 2}^{\prime}$, but the masslesss mode of these form factors are simply part of the geometric sum of mass insertions that make up this shifted pole and therefore do not effect the identification of the soft mass. There are also supersymmetric Dirac masses, associated with the Kaluza Klein modes of the gauginos between the fermions in $\Phi$ and those of $V$ i.e. $\partial_{z} \lambda \partial_{z} \chi$. In particular the field $\Phi$ is chosen to have negative parity on at least the UV or IR brane so that a massless fermion $\chi_{0}$ does not appear in the spectrum. 
The sfermion masses are given by

$$
m_{\tilde{f}}^{2}=-\left(4 \pi \alpha_{5 d}\right)^{2} \int \frac{d^{4} p}{(2 \pi)^{4}} G\left(p^{2} ; L_{0}, z\right) G\left(p^{2} ; z, L_{0}\right) p^{2} \Omega\left(\frac{p^{2}}{M^{2}}, \frac{R}{z}\right) .
$$

where we take $z=L_{1}$. It is clear that the sfermion masses will be suppressed from the intermediate resonances.

\subsubsection{Soft masses with a minimal messenger sector}

If one were to take a minimal messenger model $W=X \Phi \tilde{\Phi}$ to evaluate the current correlators then one obtains in the limit $m_{n} \ll M$

$$
\Omega\left(\frac{p^{2}}{M^{2}}, \frac{R}{z}\right)=-\frac{1}{(4 \pi)^{2}} \frac{2}{3}\left|\frac{F}{M^{2}}\right|^{2} h(x)
$$

[10] which is independent of $p^{2}(h(x) \sim 1)$. In this case the sfermion masses are given by

$$
m_{\tilde{f}}^{2} \sim \frac{2}{3}\left(\frac{\alpha_{5 d}}{4 \pi}\right)^{2}\left(\frac{R}{z}\right)^{2}\left|\frac{F}{M}\right|^{2}\left|\frac{1}{\hat{M}}\right|^{2} \times \int d p p^{5} G\left(p^{2} ; L_{0}, z\right) G\left(p^{2} ; z, L_{0}\right)
$$

Written in this way, one can see the contribution directly from dimensional analysis and the part associated with the bulk propagator/form factor associated with the resonances. In principle the supersymmetry breaking is located at $z=L_{1}$ however we have left it as a free variable in the above expression. The integral will supply a scale $m_{k k}$ after a change in varables in $p$. It is not completely free, however as the correlators have been expanded in a particular limit. Following the intuition of Hybrid mediation [36], one could change the scaling behaviour by adjusting $m_{n}, M$ and also the location along $z$ of the supersymmetry breaking closer to the UV boundary and obtain an expression of the form

$$
m_{\tilde{f}}^{2} \sim \frac{2}{3}\left(\frac{\alpha_{5 d}}{4 \pi}\right)^{2}\left(\frac{R}{z}\right)^{\tau}\left|\frac{F}{M}\right|^{2}\left|\frac{1}{\hat{M}}\right|^{\tau} \times \int d p p^{5} G\left(p^{2} ; L_{0}, z\right) G\left(p^{2} ; z, L_{0}\right)
$$

where $\tau \in[0,2] . \tau$ is actually a function of all the scales and there is an important leading coefficient, but none the less it seems promising that one could obtain a Hybrid regime where effectively only the first few resonances contribute ${ }^{8}$ and $\tau \sim 1$.

In the limit in which $m_{n} \gg M$ only the massless modes mediate supersymmetry breaking and the soft masses are given by

$$
m_{\tilde{f}}^{2}=2\left(\frac{\alpha_{5 d}}{4 \pi}\right)^{2}\left|\frac{F}{M}\right|^{2}\left(\frac{R}{z}\right)^{2}
$$

where again $z$ is set to the position of the effect action $z=L_{1}$. In all cases the gaugino soft mass terms are given by

$$
m_{\lambda_{n, m}}=2 \frac{\alpha_{5 d}}{4 \pi} \frac{F}{M}\left(\frac{R}{z}\right) f_{n} f_{m} g(x)
$$

where for $x=F / M^{2}$ and for $x<1, g(x) \sim 1$.

\footnotetext{
${ }^{8} \mathrm{~A}$ two site quiver is always "flat". In Deconstructed AdS models [72, 73] one requires at least 3 sites and therefore two resonances of $A_{\mu, i} i=1,2,3$. This may suffice as a reasonable model of the full setup.
} 
In summary, within this type of construction soft masses are completely calculable. For heavy resonances $m_{n} \gg M$ the model encodes a natural hierarchy of scales relative the $M_{P l}$, however the soft masses and scattering cross sections will appear four dimensional and the effect of resonances will be limited. Conversely in the limits $m_{n} \sim M$ and $m_{n} \ll M$ sfermion mases will be suppressed and gaugino masses unchanged.

\subsubsection{Cross sections}

Additionally it is interesting to see the effect upon scattering cross sections [20]. The form factor is associated with the bulk to boundary propagator [74]. Taking a minimal model of the supersymmetry breaking sector we look at the $e^{+} e^{-} \rightarrow\left\{\gamma_{n}\right\} \rightarrow$ hidden sector matter, where one must consider all $(n)$ photon resonances as intermediate states. This corresponds to computing $\tilde{C}_{1}(s)$. The corresponding matrix element is given by ${ }^{9}$

$$
i \mathcal{M}\left(e^{+} e-\rightarrow e^{+} e^{-}\right)=(-i e)^{2} \bar{u}(k) \gamma_{\mu} v\left(k_{+}\right) \frac{-i}{s} F(s)\left(i \tilde{C}_{1}^{\prime}(s)\right) \frac{-i}{s} F^{*}(s) \bar{v}\left(k_{+}\right) \gamma_{\nu} u(k)
$$

with $F(s)$ being the corresponding form factor. One then applies the optical theorem which acts on $\tilde{C}_{1}^{\prime}(s)$. It turns out that form factors in AdS are more naturally formulated in terms of the holographic basis instead of the Kaluza Klein basis, the specific details may be found in [70] but essentially the form factor is given by an amputated Green's function or equivalently a bulk to boundary propagator. The form factor of this model is given by taking the bulk to boundary propagator

$$
K\left(p^{2}, z^{\prime}\right)=g_{5} \sum_{n} \frac{F_{n} \psi_{n}(z)}{p^{2}-m_{n}^{2}}
$$

This encodes a sum of monopole contributions of an infinite tower of vector mesons with decay constants $F_{n}=\left\langle 0|\mathcal{O}| \rho_{n}\right\rangle$ for the nth meson of mass $m_{n}$. Defining

$$
F\left(p^{2}\right)=\int d z a(z) K(p, z) \phi(z) \tilde{\phi}(z)
$$

where $\phi(z), \tilde{\phi}(z)$ are the wavefunctions of the messenger fields (which in this case are trivial and include a $\delta\left(z-L_{1}\right)$. In general one may define

$$
g_{\rho \phi \tilde{\phi}}=\int d z a(z) \psi(z) \phi(z) \tilde{\phi}(z)
$$

in analogy to pion scattering. The size of the messenger field's charge distribution is defined from this form factor:

$$
\left\langle r^{2}\right\rangle=6 \frac{\partial}{\partial s} F(s)_{s=0}=-\sum_{n} \frac{6 g_{5} F_{n} \psi\left(L_{1}\right)}{m_{n}^{4}} .
$$

It may be evaluated by taking $F_{n}=F, \psi_{n}\left(L_{1}\right)=d(-1)^{n}$ and $m_{n}^{4}=n^{4} m_{\rho}^{4}$ to obtain

$$
\left\langle r^{2}\right\rangle=\frac{42 g_{5} F d \pi^{4}}{720 m_{\rho}^{4}}
$$

\footnotetext{
${ }^{9}$ To be compared with page 616 Chaper 18 of [75].
} 
as $F_{n} \sim m_{n}^{2} / g_{5},\left\langle r^{2}\right\rangle$ is $O\left(1 / m_{\rho}^{2}\right)$ and can be compared with eq. (5.16). The two site quiver model of section 5 has some universal couplings associated with a hidden local symmetry as observed in [57]. These slice of AdS models do not reproduce the full set of relations between $g_{\rho \phi \tilde{\phi}}$ and $g_{\rho \rho \rho}$ however they do relate separately for $g_{V \phi \tilde{\phi}}$ and for $g_{V V V}$.

Following [20] one may take a minimal messenger sector where the cross section is given by

$$
\begin{aligned}
\sigma_{1}\left(e^{+}, e^{-} \rightarrow \text { hid }\right)= & |F(s)|^{2} \frac{\left(4 \pi \alpha_{5 d}\right)^{2}}{48 \pi s^{2}} \\
& \times\left[\left(s-4 m_{+}^{2}\right)^{2} \lambda^{1 / 2}\left(s, m_{+}, m_{+}\right)+\left(m_{+} \rightarrow m_{-}\right)\right. \\
& \left.+4\left(s+2 m_{0}\right) \lambda^{1 / 2}\left(s, m_{0}, m_{0}\right)\right]
\end{aligned}
$$

where this result is related to the $\tilde{C}_{1}^{\prime}(s)$ correlator at $z^{\prime}=L_{1}$. In this way the effect of intermediate resonances are quantified and additionally the cross sections obtain a scaling behaviour. Similar examples can be found for holographic models of pion scattering [76].

\subsubsection{Messengers in the bulk}

One may imagine a situation where, for definiteness, the messenger fields $\Phi, \tilde{\Phi}$ live in the bulk and are associated with positive parity component of bulk hypermultiplets. Using the loose analogy of AdS/QCD models, where the pions are modelled by a bulk adjoint field $X\left(x_{\mu}, z\right)$ [74]. Due to the warping we expect these fields to be mostly localised towards the IR, quantified by the value $c$ of their five dimensional wavefunction $\Phi_{n}(z, c)[71]$ and on the four dimensional side of the correspondence eq. (8.1), there will be operators which correspond to these bulk fields. The supersymmetry breaking currents $\mathcal{J}(z, x)$ are extracted from their kinetic terms.

In this case we must integrate over $z$, so we may pull this factor out by dimensional analysis

$$
\Omega\left(\frac{p^{2}}{M^{2}}, \frac{R}{z}\right)=g(z) \Omega\left(\frac{p^{2}}{M^{2}}\right)
$$

As the currents are loops of messenger fields they will now be position dependent and also preserve incoming and outgoing $p_{5}$ momenta. The sfermion mass formula is then given by

$$
m_{\phi}^{2}=-\sum_{n}\left(4 \pi \alpha_{5 d}\right)^{2} \int \frac{d^{4} p}{(2 \pi)^{4}} \frac{f_{n}^{(2)}(0) f_{n}^{(2)}(0) p^{2}}{\left(p^{2}+m_{n}^{2}\right)^{2}} \Omega\left(\frac{p^{2}}{M^{2}}\right) \int f_{n}^{(2)}(z) f_{n}^{(2)}(z) g(z) d z
$$

It should be clear that even without specifying the exact form of the current correlators the leading sfermion mass contribution will be suppressed. The relevant cross sections of section 6 will involve a single summation over resonances instead of a double sum. This single sum instead of double sum would appear in cross sections too, as an interference effect, essentially pulling out the sum on $n$ in the form factor $\sum_{n}\left|F_{n}(q)\right|^{2}$.

Finally, if we located the correlators in the UV $\tilde{C}_{a}\left(z=L_{0}\right)$ then only the massless modes, whose wavefunctions are flat, would mediate the supersymmetry breaking at all. This corresponds to a limit in which the external field no longer mixes with the resonances. 


\subsection{Computing correlators of $\mathcal{O}$}

There are many operators represented by $\mathcal{O}$. These currents are associated with the broken CFT or AdS background [66] equivalent to the bulk gauge field self couplings [67, 77, 78], where essentially resonances of $V_{n}$ and $\Phi_{A d j, n}$ run in the loop, and the coupling of the gauge field to other bulk fields. All these will generate contributions to the one loop vacuum polarisation amplitude of the external gauge field and therefore to the beta function, $\Delta b_{b u l k}$, or running gauge coupling of the external field. The effective action associated with these contributions will be analogous to eq. (8.18) but located at the UV boundary. The "supertraced" combination eq. (2.7) of this current multiplet vanishes [3] however, as they contain no supersymmetry breaking effects ${ }^{10}$ within this type of supersymmetry breaking. The supersymmetry breaking affects are associated with the $J$ multiplet located at $z=L_{1}$, which is still a one loop correction to the external gauge field but which first descends down to couple to the relevant operators. This is much the same as for lepton annihilation to pions in models of AdS/QCD. The experimentally observed vacuum polarisation amplitude that couples to the external fields is to all orders in the hidden sector and that includes the contributions from currents contained in $\mathcal{O}, \mathbb{J}$ and $J$. Such an effective action could be achieved by completely integrating out the IR and bulk generating functionals $Z_{\text {AdS }}[\mathcal{O}] \times$ $Z_{\mathrm{IR}}[J]$, leaving only

$$
Z_{\mathrm{SSM}}[\mathbb{J}] \times e^{i S_{\mathrm{eff}}\left(V_{0}\right)}
$$

\subsection{A smoother transition?}

Finally let us discuss an application of Holography closer to the soft wall or unparticle scenario, where instead of an IR brane cutoff we wish for a smoother regulator associated with a $z$ dependent dilaton profile [79]. We can then imagine locating the supersymmetry breaking peaked at some definite value of $z=L_{1}$. First setting the gauge couplings to zero, the propagators of the gauge fields are determined entirely by the geometry and their coupling to the dilaton. Switching the gauge coupling back on, we can work perturbatively to generate suitable soft masses. To obtain the results for a soft wall model from the above results, one may simply replace $G\left(z, z^{\prime}\right)$ with that obtained from the soft wall equations of motion.

\section{Discussion and conclusion}

In this paper we have explored a natural extension of general gauge mediation to cases where the mediating gauge fields, instead of having a two point function of a free theory, have a two point function described by a non trivial spectral function. We have shown that this covers a very broad class of models, including those that involve a strongly coupled hidden sector, approximate conformality or Kaluza-Klein modes associated with an extra dimension. Despite covering a broad class of models some general features may be extracted: even within entirely four dimensional models of a strongly coupled hidden

\footnotetext{
${ }^{10} \mathrm{Up}$ to two loop order in the gauge coupling. There are three loop (and higher) contributions for instance as all the Gaugino (and kk mode) poles will be shift by a soft mass [24].
} 
sector, it is likely that one obtains suppressed scalar soft masses relative to gaugino masses at the high scale $M$ before RG running. This is inferred by assuming that some hidden sector resonances will share the gauge field quantum numbers of the standard model and then vector meson dominance may ensue. Of course these results are dependent on the assumption of a generalised form of vector meson dominance and we are mindful to point out that not all strongly coupled models exhibit this phenomena: some models may have straightforward magnetic duals.

However within these models, typically one must compare the resonance scale with the scale $M$ of the hidden sector and for a sufficiently low resonance scale sfermion masses are suppressed at leading order and one observes a scaling in the cross sections of visible matter to hidden sector messenger fields.

It seems that to obtain quantitive predictions for effects on scattering cross sections and soft masses, particularly from string models, it may be useful to simply integrate out $[80,81]$ all the higher states and extract the lowest resonance masses and relevant form factors. An interesting consequence of this is that, if one starts from the holographic model above and integrates out all the higher resonances one may hope to arrive at a quiver model very similar to that obtained starting from the magnetic description of certain Seiberg dual models explored in [9, 11, 36, 42].

We also identified for the simplest quiver the "supercurrent field identity" and "modified current operator" which are useful tools for interpreting resonances as they are part of the vector dominance model and showing how these are related to the operator-field correspondence of the AdS/CFT perspective. In a general sense these resonance mediation models capture the same features that were found with photon interactions to hadrons, so it is rather appealing and well motivated to build these features in to gauge mediation. We believe that this is a powerful confirmation of this approach.

One point we hope to have emphasised is that the general gauge mediation programme [3] is not simply a parameterisation of soft terms of the MSSM, it also encodes scattering cross sections [20] of visible $\rightarrow$ hidden. In this paper we have extended these cross sections to general four dimensional strongly coupled models and five dimensional models, in which there are intermediate resonances. In particular the use of different types of integrals of the cross sections have been explored, to in principle reveal Schwinger terms as signs of vector meson dominance. It would be interesting to extend this work to hidden sector $\rightarrow$ hidden sector scattering: whilst most of the examples in this paper have focused on AdS/CFT type constructions one can't help but feel that analysing the current correlators and scattering cross sections using Regge theory [46] may be as worthwhile.

\section{Acknowledgments}

MM is funded by the Alexander von Humboldt Foundation. MM would like to thank Felix Brummer, Andreas Weiler, Mathias Garny, Daniel C. Thompson and Alberto Mariotti for fruitful discussions. The diagrams are drawn in JaxoDraw $[82,83]$. 
Open Access. This article is distributed under the terms of the Creative Commons Attribution License which permits any use, distribution and reproduction in any medium, provided the original author(s) and source are credited.

\section{References}

[1] G. Giudice and R. Rattazzi, Theories with gauge mediated supersymmetry breaking, Phys. Rept. 322 (1999) 419 [hep-ph/9801271] [INSPIRE].

[2] E. Witten, Dynamical breaking of supersymmetry, Nucl. Phys. B 188 (1981) 513 [InSPIRE].

[3] P. Meade, N. Seiberg and D. Shih, General gauge mediation, Prog. Theor. Phys. Suppl. 177 (2009) 143 [arXiv:0801.3278] [INSPIRE].

[4] M. Buican, P. Meade, N. Seiberg and D. Shih, Exploring general gauge mediation, JHEP 03 (2009) 016 [arXiv:0812.3668] [INSPIRE].

[5] D. Marques, Generalized messenger sector for gauge mediation of supersymmetry breaking and the soft spectrum, JHEP 03 (2009) 038 [arXiv:0901.1326] [INSPIRE].

[6] F. Galli and A. Mariotti, Soft Spectrum in Yukawa-Gauge Mediation, JHEP 06 (2012) 055 [arXiv: 1201.4893] [INSPIRE].

[7] E.A. Mirabelli and M.E. Peskin, Transmission of supersymmetry breaking from a four-dimensional boundary, Phys. Rev. D 58 (1998) 065002 [hep-th/9712214] [INSPIRE].

[8] M. McGarrie and D.C. Thompson, Warped general gauge mediation, Phys. Rev. D 82 (2010) 125034 [arXiv:1009.4696] [INSPIRE].

[9] M. McGarrie, Hybrid gauge mediation, JHEP 09 (2011) 138 [arXiv:1101.5158] [INSPIRE].

[10] M. McGarrie and R. Russo, General gauge mediation in 5D, Phys. Rev. D 82 (2010) 035001 [arXiv: 1004.3305] [INSPIRE].

[11] M. McGarrie, General gauge mediation and deconstruction, JHEP 11 (2010) 152 [arXiv: 1009.0012] [INSPIRE].

[12] M. McGarrie, Gauge mediated supersymmetry breaking in five dimensions, arXiv:1109.6245 [INSPIRE].

[13] T. Bauer, R. Spital, D. Yennie and F. Pipkin, The hadronic properties of the photon in high-energy interactions, Rev. Mod. Phys. 50 (1978) 261 [Erratum ibid. 51 (1979) 407] [INSPIRE].

[14] F.J. Gilman, Photoproduction and electroproduction, Phys. Rept. 4 (1972) 95 [INSPIRE].

[15] R. Feynman, Photon-hadron interactions, WA Benjamin, Inc., Reading U.S.A. (1972).

[16] S.P. Martin, Generalized messengers of supersymmetry breaking and the sparticle mass spectrum, Phys. Rev. D 55 (1997) 3177 [hep-ph/9608224] [INSPIRE].

[17] C. Kilic, T. Okui and R. Sundrum, Colored resonances at the Tevatron: phenomenology and discovery potential in multijets, JHEP 07 (2008) 038 [arXiv:0802.2568] [INSPIRE].

[18] C. Kilic, T. Okui and R. Sundrum, Vectorlike confinement at the LHC, JHEP 02 (2010) 018 [arXiv: 0906.0577] [INSPIRE].

[19] J. Sakurai, Duality in $e^{+} e^{-} \rightarrow$ hadrons?, Phys. Lett. B 46 (1973) 207 [InSPIRE].

[20] J.-F. Fortin, K. Intriligator and A. Stergiou, Superconformally covariant OPE and general gauge mediation, JHEP 12 (2011) 064 [arXiv:1109.4940] [INSPIRE]. 
[21] D. Son and M. Stephanov, $Q C D$ and dimensional deconstruction, Phys. Rev. D 69 (2004) 065020 [hep-ph/0304182] [InSPIRE].

[22] R. Sundrum, From fixed points to the fifth dimension, Phys. Rev. D 86 (2012) 085025 [arXiv: 1106.4501] [INSPIRE].

[23] N. Kroll, T. Lee and B. Zumino, Neutral vector mesons and the hadronic electromagnetic current, Phys. Rev. 157 (1967) 1376 [rNSPIRE].

[24] J.Y. Lee, Renormalization in general gauge mediation, JHEP 10 (2010) 041 [arXiv: 1001.1940] [INSPIRE].

[25] R. Argurio, M. Bertolini, G. Ferretti and A. Mariotti, Patterns of soft masses from general semi-direct gauge mediation, JHEP 03 (2010) 008 [arXiv:0912.0743] [INSPIRE].

[26] T. Lee, S. Weinberg and B. Zumino, Algebra of fields, Phys. Rev. Lett. 18 (1967) 1029 [INSPIRE].

[27] K. Kang, Field-current identities, broken symmetries, current-mixing model and the algebra of gauge fields, Phys. Rev. 177 (1969) 2439 [INSPIRE].

[28] J. Sakurai, Currents and mesons, University of Chicago press, Chicago U.S.A. (1969).

[29] T. Lee and B. Zumino, Field current identities and algebra of fields, Phys. Rev. 163 (1967) 1667 [INSPIRE].

[30] M. Bando, T. Kugo, S. Uehara, K. Yamawaki and T. Yanagida, Is $\rho$ meson a dynamical gauge boson of hidden local symmetry?, Phys. Rev. Lett. 54 (1985) 1215 [INSPIRE].

[31] M. Gell-Mann, A schematic model of baryons and mesons, Phys. Lett. 8 (1964) 214 [INSPIRE].

[32] Y. Nambu, Possible existence of a heavy neutral meson, Phys. Rev. 106 (1957) 1366 [INSPIRE].

[33] M. Gell-Mann and F. Zachariasen, Form-factors and vector mesons, Phys. Rev. 124 (1961) 953 [INSPIRE].

[34] Y. Nambu and J. Sakurai, Rare decay modes of the $\omega(\eta)$ meson, Phys. Rev. Lett. 8 (1962) 79 [inSPIRE].

[35] M. Sudano, General gaugino mediation, arXiv:1009.2086 [INSPIRE].

[36] R. Auzzi and A. Giveon, The sparticle spectrum in minimal gaugino-gauge mediation, JHEP 10 (2010) 088 [arXiv: 1009.1714] [INSPIRE].

[37] R. Auzzi, A. Giveon and S.B. Gudnason, Flavor of quiver-like realizations of effective supersymmetry, JHEP 02 (2012) 069 [arXiv:1112.6261] [INSPIRE].

[38] R. Auzzi, A. Giveon and S.B. Gudnason, Mediation of supersymmetry breaking in quivers, JHEP 12 (2011) 016 [arXiv:1110.1453] [INSPIRE].

[39] R. Auzzi, A. Giveon, S.B. Gudnason and T. Shacham, On the spectrum of direct gaugino mediation, JHEP 09 (2011) 108 [arXiv:1107.1414] [INSPIRE].

[40] R. Auzzi and A. Giveon, Superpartner spectrum of minimal gaugino-gauge mediation, JHEP 01 (2011) 003 [arXiv:1011.1664] [INSPIRE].

[41] M.A. Shifman, A. Vainshtein and V.I. Zakharov, QCD and resonance physics: applications, Nucl. Phys. B 147 (1979) 448 [inSPIRE].

[42] D. Green, A. Katz and Z. Komargodski, Direct gaugino mediation, Phys. Rev. Lett. 106 (2011) 061801 [arXiv: 1008.2215] [INSPIRE]. 
[43] K.R. Dienes, E. Dudas and T. Gherghetta, Grand unification at intermediate mass scales through extra dimensions, Nucl. Phys. B 537 (1999) 47 [hep-ph/9806292] [INSPIRE].

[44] E. Ruiz Arriola and W. Broniowski, Pion electromagnetic form factor, perturbative QCD and large- $N_{c}$ Regge models, Phys. Rev. D 78 (2008) 034031 [arXiv:0807.3488] [InSPIRE].

[45] C. Dominguez, Electromagnetic form factors of hadrons in dual-large- $N_{c} Q C D$, AIP Conf. Proc. 1361 (2011) 182 [arXiv:1002.3720] [INSPIRE].

[46] P. Collins, An introduction to regge theory and high-energy physics, Cambridge University Press, Cambridge U.K. (1977).

[47] C. Dominguez and T. Thapedi, Electromagnetic proton form factors in large- $N_{c} Q C D$, JHEP 10 (2004) 003 [hep-ph/0409111] [INSPIRE].

[48] J. Cumming and H. Osborn, Hadronic interactions of electrons and photons, Academic Press Inc, U.S.A. (1971).

[49] A. Karch, E. Katz, D.T. Son and M.A. Stephanov, Linear confinement and AdS/QCD, Phys. Rev. D 74 (2006) 015005 [hep-ph/0602229] [INSPIRE].

[50] Z. Abidin and C.E. Carlson, Nucleon electromagnetic and gravitational form factors from holography, Phys. Rev. D 79 (2009) 115003 [arXiv: 0903.4818] [INSPIRE].

[51] H. Georgi, Unparticle physics, Phys. Rev. Lett. 98 (2007) 221601 [hep-ph/0703260] [INSPIRE].

[52] M. Stephanov, Deconstruction of unparticles, Phys. Rev. D 76 (2007) 035008 [arXiv: 0705.3049] [INSPIRE].

[53] G. Cacciapaglia, G. Marandella and J. Terning, The AdS/CFT/unparticle correspondence, JHEP 02 (2009) 049 [arXiv: 0804.0424] [INSPIRE].

[54] J. Bagger and C. Xiong, AdS $S_{5}$ supersymmetry in $N=1$ superspace, JHEP 07 (2011) 119 [arXiv: 1105.4852] [INSPIRE].

[55] D. Marti and A. Pomarol, Supersymmetric theories with compact extra dimensions in $N=1$ superfields, Phys. Rev. D 64 (2001) 105025 [hep-th/0106256] [INSPIRE].

[56] D. Schildknecht, Vector meson dominance, photo- and electroproduction from nucleons, Springer Tracts Mod. Phys. 63 (1972) 57.

[57] Z. Komargodski, Vector mesons and an interpretation of Seiberg duality, JHEP 02 (2011) 019 [arXiv: 1010.4105] [INSPIRE].

[58] C. Itzykson and J. Zuber, Quantum field theory, Dover Publications, U.S.A. (1980).

[59] A. Bramon, E. Etim and M. Greco, A vector meson dominance approach to scale invariance, Phys. Lett. B 41 (1972) 609 [InSPIRE].

[60] J. van der Bij and M. Veltman, Two loop large Higgs mass correction to the $\rho$ parameter, Nucl. Phys. B 231 (1984) 205 [InSPIRE].

[61] A. Ghinculov and J. van der Bij, Massive two loop diagrams: the Higgs propagator, Nucl. Phys. B 436 (1995) 30 [hep-ph/9405418] [InSPIRE].

[62] N. Craig, S. Knapen, D. Shih and Y. Zhao, A complete model of low-scale gauge mediation, arXiv: 1206.4086 [INSPIRE].

[63] M. Rho, Chiral nuclear dynamics: From quarks to nuclei to compact stars, World Scientific Publishing Company, Singapore (2008).

[64] Y. Nomura, Supersymmetric unification in warped space, hep-ph/0410348 [INSPIRE]. 
[65] A. Hebecker, $5 D$ super Yang-Mills theory in $4 D$ superspace, superfield brane operators and applications to orbifold GUTs, Nucl. Phys. B 632 (2002) 101 [hep-ph/0112230] [INSPIRE].

[66] N. Arkani-Hamed, M. Porrati and L. Randall, Holography and phenomenology, JHEP 08 (2001) 017 [hep-th/0012148] [INSPIRE].

[67] L. Randall and M.D. Schwartz, Quantum field theory and unification in $A d S_{5}$, JHEP 11 (2001) 003 [hep-th/0108114] [INSPIRE].

[68] K. Agashe and A. Delgado, A note on CFT dual of RS model with gauge fields in bulk, Phys. Rev. D 67 (2003) 046003 [hep-th/0209212] [InSPIRE].

[69] Z. Chacko and E. Ponton, Bulk gauge fields in warped space and localized supersymmetry breaking, JHEP 11 (2003) 024 [hep-ph/0301171] [INSPIRE].

[70] M. McGarrie, Holography for general gauge mediation, JHEP 02 (2013) 132 [arXiv: 1210.4935] [INSPIRE].

[71] T. Gherghetta and A. Pomarol, Bulk fields and supersymmetry in a slice of AdS, Nucl. Phys. B 586 (2000) 141 [hep-ph/0003129] [INSPIRE].

[72] J. de Blas, A. Falkowski, M. Pérez-Victoria and S. Pokorski, Tools for deconstructing gauge theories in $A d S_{5}$, JHEP 08 (2006) 061 [hep-th/0605150] [INSPIRE].

[73] A. Falkowski and M. Pérez-Victoria, Holography, pade approximants and deconstruction, JHEP 02 (2007) 086 [hep-ph/0610326] [INSPIRE].

[74] J. Erlich, E. Katz, D.T. Son and M.A. Stephanov, QCD and a holographic model of hadrons, Phys. Rev. Lett. 95 (2005) 261602 [hep-ph/0501128] [INSPIRE].

[75] M.E. Peskin and D.V. Schroeder, An introduction to quantum field theory, Westriew Press, U.S.A. (1995).

[76] L. Da Rold and A. Pomarol, Chiral symmetry breaking from five dimensional spaces, PoS (HEP2005) 355.

[77] W.D. Goldberger and I.Z. Rothstein, High-energy field theory in truncated AdS backgrounds, Phys. Rev. Lett. 89 (2002) 131601 [hep-th/0204160] [INSPIRE].

[78] L. Randall and M.D. Schwartz, Unification and the hierarchy from AdS5, Phys. Rev. Lett. 88 (2002) 081801 [hep-th/0108115] [INSPIRE].

[79] A. Rajaraman, On the decay of unparticles, Phys. Lett. B 671 (2009) 411 [arXiv:0806.1533] [inSPIRE].

[80] J. Hirn and V. Sanz, Interpolating between low and high energy QCD via a $5 D$ Yang-Mills model, JHEP 12 (2005) 030 [hep-ph/0507049] [INSPIRE].

[81] M. Harada, S. Matsuzaki and K. Yamawaki, Holographic QCD integrated back to hidden local symmetry, Phys. Rev. D 82 (2010) 076010 [arXiv:1007.4715] [InSPIRE].

[82] D. Binosi and L. Theussl, JaxoDraw: a graphical user interface for drawing Feynman diagrams, Comput. Phys. Commun. 161 (2004) 76 [hep-ph/0309015] [INSPIRE].

[83] D. Binosi, J. Collins, C. Kaufhold and L. Theussl, JaxoDraw: a graphical user interface for drawing Feynman diagrams. Version 2.0 release notes, Comput. Phys. Commun. 180 (2009) 1709 [arXiv:0811.4113] [INSPIRE]. 